\title{
Adaptive Disturbance Observer for Trajectory Tracking of Underwater Vehicles
}

\author{
J. Guerrero ${ }^{\mathrm{a}}$, J. Torres ${ }^{\mathrm{a}}$, V. Creuze ${ }^{\mathrm{b}}$, A. Chemori $^{\mathrm{b}}$ \\ ${ }^{a}$ Center for Research and Advanced Studies of the National Polytechnic Institute \\ (CINVESTAV), Automatic Control Department, Mexico City, MX, 07360,(e-mail: \\ \{jguerrero, jtorres\}@ctrl.cinvestav.mx). \\ ${ }^{b}$ LIRMM, Univ. Montpellier, CNRS, Montpellier, France (e-mail: \{vincent.creuze, \\ ahmed.chemori\}@lirmm.fr).
}

\begin{abstract}
Complex and highly coupled dynamics, time-variance, unpredictable disturbances and lack of knowledge of hydrodynamic parameters, complicate the control of underwater vehicles. This paper deals with the adaptive disturbance observer design for the robust trajectory tracking problem for underwater vehicles in presence of unknown external disturbances and parametric uncertainties. First, the dynamics of the vehicle is transformed into the socalled regular form. Then, based on the Extended State Observer technique and High Order Sliding Modes Control, a disturbance observer is proposed. Furthermore, the gains of the observer are automatically adjusted by the introduction of an adaption law. The stability of the whole controller/observer scheme is proven using Lyapunov's arguments. The adaptive disturbance observer aims to improve the Backstepping and nonlinear PD controllers. Real-Time experiments demonstrate the effectiveness of the proposed algorithm for the trajectory tracking task under several scenarios.
\end{abstract}

Keywords: Sliding Mode Control, Backstepping, PD, Autonomous Underwater Vehicles, Adaptive Control

\section{Introduction}

The Remotely Operated Vehicles (ROVs) and Autonomous Underwaters 3 Vehicles (AUVs) have been an invaluable tool for research marine environ4 ment. These two classes of underwater vehicles has proven their value in 5 a wide range of applications, such as inspection, exploration, oceanography, 
biology, and so on. In some applications, the vehicle's autonomy plays a fundamental role in the success of the mission. Thus, to provide autonomy to underwater vehicles, there are three main tasks to perform, namely: 1) Station keeping, which refers to maintaining the vehicle to constant position and attitude; 2) Path following which is the task where the vehicle follows a spatial reference; and 3) Trajectory tracking, which means that the vehicle follows a time varying trajectory. In this paper, we focus on the first and third cases.

The design of a trajectory tracking controller for an underwater vehicle is not trivial. The control of underwater vehicles is challenging due to the non-linearity, time-variance, random external disturbances, such as the environmental force generated by the sea current fluctuation, and the difficulty in accurately modeling hydrodynamic effects [1].

Several strategies have been proposed to control underwater vehicles, such as Proportional-Derivative (PD) control [2, 3], Proportional Integral Derivative (PID) control [4, 5], $H_{2}$ and $H_{\infty}$ control [6], Optimal Control [7], to name a few. Moreover, many controllers were designed employing the linearized model of underwater vehicles, considering strong restrictive assumptions to simplify the mathematical description, resulting sometimes in low robustness to both external disturbances and model uncertainties. This is the reason why many researchers concentrated their interests in developing robust controllers for underwater vehicles.

The Sliding Mode Control (SMC) is a robust technique, which allows controlling the vehicle despite external disturbances and parameters uncertainties. For example, a second order sliding mode controller for trajectory tracking task for an underwater vehicle is proposed in [8]. In order to minimize the energy consumption of the controller, a Super-Twisting SMC with region concept is proposed in [9]. However, the main drawback of the SMC is the chattering effect induced by the signum function. Although other functions can replace the signum function [10], it constrains the sliding systems trajectories, not to the sliding surface but to its vicinity, thus partly losing the robustness to the disturbances [11].

Backstepping Control (BSC) is another popular technique, sometimes used to control underwater vehicles. The BSC technique offers a systematic procedure to construct the Lyapunov functions and related stabilizing feedback control laws recursively [12]. For example, a bioinspired filtered Backstepping tracking control for the kinematic model of an underwater vehicle is proposed in [13]. In [14] an error-based block BSC for the trajectory 
tracking of a submarine is proposed. However, the drawback of the BSC is that it requires perfect cancellation of the robot's nonlinear dynamics, which means that the exact knowledge of this latter is needed.

PD and PID controllers are popular techniques used to control the position and attitude if underwater vehicles due to their simple design and good performance. However, it is well-known that the performance of the PD/PID controller is degraded when the plant is highly nonlinear or time-varying. To overcome these drawbacks, the PD/PID controllers are improved adopting strategies based on auto-adjustable [15], saturated [16], adaptive [17], or nonlinear functions [18, 19]. For instance, a nonlinear PID for the trajectory tracking of an AUV is proposed in Guerrero et al. [19]. In this work, a PID was saturated by a whole set of nonlinear functions in order to provide robustness towards external disturbances and parametric uncertainties. In general, PD and PID improved controllers can preserve easy design and gain robustness towards external disturbance, as one can see on the results of the above cited papers.

The disturbance observation focuses on controller improvement through counteracting of the disturbances. For example, the Extended State Observer (ESO) methodology is applied to an AUV trajectory tracking in [20]. In this work, the authors propose an adaptive ESO algorithm to estimate the unknown submarine velocity, parametric uncertainties and external disturbances for the full six Degrees of Freedom (DoF) of the system. Then, an Integral Sliding Mode Control (ISMC) is designed and includes the disturbance estimation made by the ESO. Based on real-time experiments, the authors show the improvement of the ISMC and compare the proposed algorithm with the classical PD controller. Nonetheless, the proposed control scheme needs the adjustment of many controller gains, which can be timeconsuming. Also, the control law uses the signum function, which causes chattering, as can be seen on the reported control input graphs. Finally, although the algorithm was designed to compensate parametric uncertainties, experiments about robustness against parameter changes are not shown.

In this paper, we develop an adaptive disturbance observer based on the Generalized Super-Twisting Algorithm (GSTA) 21] through the ESO technique in order to improve robustness to both external disturbances and model uncertainties. For example, based on the real-time experiments shown in work [14], the proposed BSC shows a constant offset between the vehicle and the desired trajectory. Similarly, in the experiments that were carried out with a nonlinear PD (NLPD) control law, in the study [18, we can 
observe a constant error in steady-state behavior in the depth tracking tests when parametric uncertainties are considered. In this sense, the developed disturbance observer is introduced into BSC and NLPD control laws, in order to counteract the effects of the external disturbances and the parametric uncertainties.

The main contributions of this paper are as follows:

1. An Adaptive disturbance observer based on High Order Sliding Mode Controllers and Extended State Observer is developed to estimate and compensate the effects of external disturbances and parameters uncertainties during trajectory tracking for an underwater vehicle. Moreover, the stability analysis of the whole scheme observer/controller is proven by Lyapunov's arguments.

2. The adaptive GSTA-ESO extends our previous results (see Guerrero et al. [22]) introducing adaption laws to update the observer's gains, then relaxing the requirement of knowing the upper bound of perturbations.

3. The GSTA-ESO improves the BSC and NLPD controllers performances shown in [14] and [18], respectively.

4. The effectiveness of the proposed adaptive observer is validated through real-time experiments.

The rest of the paper is organized as follows: a brief description of the dynamics of an underwater vehicle is given in Section 2. BSC and NLPD enhancement with the adaptive disturbance observer technique are described in Section 3. The real-time experimental results for two DoF trajectory tracking are presented and analyzed in Section 4. Finally, some concluding remarks are delineated in Section 5.

\section{Dynamic Model}

The mathematical model is described with respect to an earth-fixed frame $\left(x_{I}, y_{I}, z_{I}\right)$, and a body-fixed reference frame $\left(x_{b}, y_{b}, z_{b}\right)$ as shown in Figure 1 .

The matrix of spatial transformation between the inertial frame and the frame of the rigid body can be defined through the transformation of the Euler angles, $J(\eta)$, and the following equation:

$$
\dot{\eta}=J(\eta) \nu
$$




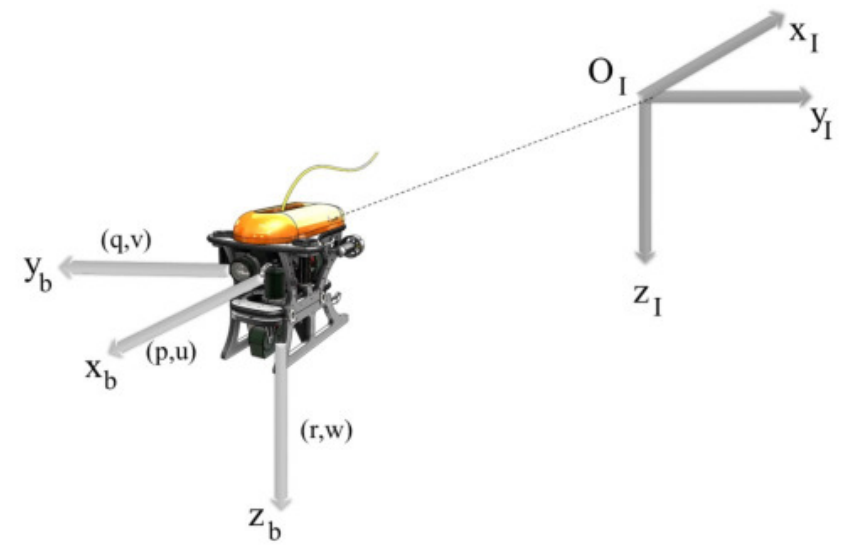

Figure 1: Leonard underwater vehicle reference frames. The earth-fixed frame is denoted $\left(O_{I}, x_{I}, y_{I}, z_{I}\right)$ and the body-fixed frame is denoted $\left(O_{b}, x_{b}, y_{b}, z_{b}\right)$.

where $\nu=[u, v, w, p, q, r]^{T}$ is the vector of velocity in the body-fixed frame and $\eta=[x, y, z, \phi, \theta, \psi]^{T}$ represents the vector of position and orientation in the earth-fixed frame. Moreover, the SNAME notation [23] is usually employed to describe the mathematical model of the underwater vehicles, which can be written as follows [4, 24, 5, 25]:

$$
M(\nu) \dot{\nu}+C(\nu) \nu+D(\nu) \nu+g(\eta)=\tau+w_{e}(t)
$$

where $M(\nu) \in \mathbb{R}^{6 \times 6}$ is the matrix of inertia (including the effects of added mass), $C(\nu) \in \mathbb{R}^{6 \times 6}$ is the Coriolis-centripetal matrix, $D(\nu) \in \mathbb{R}^{6 \times 6}$ represents the hydrodynamic damping matrix, $g(\eta) \in \mathbb{R}^{6}$ is the vector of gravitational/buoyancy forces and moments. Finally, $\tau \in \mathbb{R}^{6}$ is the control vector acting on the underwater vehicle, and $w_{e}(t) \in \mathbb{R}^{6}$ represents the vector of external disturbances.

By applying the velocity transformation mapping given by Eq. (1) to Eq. (2), it is possible to express the dynamics in the earth-fixed frame as follows (see [14, 22] for more details):

$$
\underbrace{M_{\eta}(\eta) \ddot{\eta}+C_{\eta}(\nu, \eta) \dot{\eta}+D_{\eta}(\nu, \eta) \dot{\eta}+g_{\eta}(\eta)}_{f(\eta, \nu)}=\tau_{\eta}+w_{\eta}(t)
$$

where $f(\eta, \nu)$ is the system dynamics. As highlighted by the work [4, 24], the elements of the matrices of the dynamic model (3) depend of a large set 
of parameters, difficult to estimate [26]. For this reason, it is common to introduce assumptions to reduce the number of parameters. From equation (3), the system dynamics $f(\eta, \nu)$ can be written as the sum of the estimated dynamics $\hat{f}(\eta, \nu)$ and the unknown dynamics $\tilde{f}(\eta, \nu)$, as follows:

$$
f(\eta, \nu)=\hat{f}(\eta, \nu)+\tilde{f}(\eta, \nu)
$$

where:

$$
\begin{aligned}
& \hat{f}(\eta, \nu)=\hat{M}_{\eta}(\eta) \ddot{\eta}+\hat{C}_{\eta}(\nu, \eta) \dot{\eta}+\hat{D}_{\eta}(\nu, \eta) \dot{\eta}+\hat{g}_{\eta}(\eta) \\
& \tilde{f}(\eta, \nu)=\tilde{M}_{\eta}(\eta) \ddot{\eta}+\tilde{C}_{\eta}(\nu, \eta) \dot{\eta}+\tilde{D}_{\eta}(\nu, \eta) \dot{\eta}+\tilde{g}_{\eta}(\eta)
\end{aligned}
$$

here the matrices of the unknown dynamics vector $\tilde{f}(\eta, \nu)$ are defined as $\tilde{M}_{\eta}=M_{\eta}-\hat{M}_{\eta}, \tilde{C}_{\eta}=C_{\eta}-\hat{C}_{\eta}, \tilde{D}_{\eta}=D_{\eta}-\hat{D}_{\eta}$ and $\tilde{g}_{\eta}=g_{\eta}-\hat{g}_{\eta}$.

Finally, the mathematical model of the underwater vehicle can be expressed with respect to known parameters by introducing the relation (4) into the dynamic system (3), which leads to

$$
\hat{M}_{\eta}(\eta) \ddot{\eta}+\hat{C}_{\eta}(\nu, \eta) \dot{\eta}+\hat{D}_{\eta}(\nu, \eta) \dot{\eta}+\hat{g}_{\eta}(\eta)=\tau_{\eta}+\bar{d}(t)
$$

where the lumped unknown disturbance vector is defined as $\bar{d}(t)=w_{\eta}(t)-$ $\tilde{f}(\eta, \nu)$. It is worth to note that the disturbance vector $\bar{d}(t)$ contains the effects of the external disturbance and the unknown dynamics as well.

\section{Adaptive Disturbance Observer and Trajectory Tracking Con- troller Design}

In this section, a new adaptive disturbance observer is introduced. This observer is based on the Extended State Observer technique which has been proposed originally in [27, 28]. The ESO method is applied to integral chain systems. The main idea behind the ESO technique is to design a state observer for a new augmented system, which considers the external disturbance term as an additional state. Then, the estimation made by the observer will provide information about the system's states and the external disturbance as well. The main drawback of this technique is that the bound of the external disturbance needs to be known precisely in order to obtain a proper tuning of the observer's gains. Based on this issue, in the study [29], we proposed a GSTA-ESO based on High Order Sliding Modes theory. The main advantage of the proposed method is that the estimation of the disturbance 
is made in finite time. The effectiveness of the GSTA-ESO is demonstrated through real-time experiments. The experiments show the improvement of the NLPD nominal design towards parametric uncertainties and external disturbances as well. In theory, the observer of the proposed scheme has three gains to tune, directly related to the upper bound of the disturbance. In this manuscript, we have extended the results shown in our previous work, introducing adaption laws to update the observer's gains depending on the disturbance evolution. It means that the controller will be capable of rejecting bounded time-varying disturbances even if the upper bound of the perturbation is not known.

\subsection{Adaptive Disturbance Observer Design}

First, from the vehicle's dynamics described by Eq. (7), we introduce the following state variables:

$$
\zeta_{1}(t)=\eta(t) \quad ; \zeta_{2}(t)=\dot{\eta}(t)
$$

Then, the mathematical model, (7), can be rewritten into the so-called regular form as follows:

$$
\begin{aligned}
& \dot{\zeta}_{1}(t)=\zeta_{2}(t) \\
& \dot{\zeta}_{2}(t)=\bar{F}(\zeta)+G(\zeta) \tau_{\eta}+d(t)
\end{aligned}
$$

where:

$$
\begin{aligned}
\bar{F}(\zeta) & =-\hat{M}_{n}(\eta)^{-1}\left[\hat{C}_{n}(\nu, \eta) \dot{\eta}+\hat{D}_{\eta}(\nu, \eta) \dot{\eta}+\hat{g}_{\eta}(\eta)\right] \\
G(\zeta) & =\hat{M}_{n}(\eta)^{-1} \\
d(t) & =\hat{M}_{\eta}(\eta)^{-1} \bar{d}(t)
\end{aligned}
$$

Before proposing the adaptive disturbance observer, we need to introduce the following assumptions:

Assumption 1. The pitch angle is smaller than $\pi / 2$, i.e., $|\theta|<\pi / 2$.

Assumption 2. The external disturbance $d(t)$ is a Lipschitz continuous signal, but its upper bound is not necessarily known. 
According to Assumption 1, the inverse of the matrix $J(\eta)$ exists. Also, according to Assumption 2, the time derivative of the lumped external disturbance terms $d(t)$ exists almost everywhere and it is bounded:

$$
\left|\dot{d}_{i}(t)\right| \leq L_{i}, \quad i=\overline{1,6}
$$

152

In this work, we assume that the waves and currents fulfill Assumption 2.

The first step of the design of the adaptive disturbance observer is to introduce the next auxiliary variable $\sigma(t)$ defined as:

$$
\sigma(t)=\zeta_{2}(t)+\Lambda \zeta_{1}(t)
$$

where $\sigma(t):=\left[\sigma_{1}, \sigma_{2}, \cdots, \sigma_{6}\right]^{T}$ and $\Lambda=\operatorname{diag}\left(\lambda_{1}, \lambda_{2}, \cdots, \lambda_{6}\right)$ is a diagonal, and positive definite matrix.

Computing the time derivative of (11) leads to:

$$
\dot{\sigma}(t)=F(\zeta)+G(\zeta) \tau_{\eta}+d(t)
$$

with $F(\zeta)=\bar{F}(\zeta)+\Lambda \dot{\zeta}_{1}(t)$

The second step is to consider the total disturbance $d(t)$ in Eq. (12) as an extended state $\chi(t)$ as follows:

$$
\begin{aligned}
& \dot{\sigma}(t)=F(\zeta)+G(\zeta) \tau_{\eta}+\chi(t) \\
& \dot{\chi}(t)=h(t)
\end{aligned}
$$

where $h(t)$ is the time derivative of the total disturbance $d(t)$.

Remark 1. It is worth to note that the time derivative of the disturbance, $h(t)$, is used to design the disturbance observer only. This parameter will be estimated by the adaptive algorithm.

Finally, we propose a new disturbance observer which is constructed as follows:

$$
\begin{aligned}
\tilde{\sigma}(t) & =\hat{\sigma}(t)-\sigma(t) \\
\tilde{\chi}(t) & =\hat{\chi}(t)-\chi(t) \\
\dot{\hat{\sigma}} & =F(\zeta)+G(\zeta) \tau_{\eta}-K_{1} \Phi_{1}(\tilde{\sigma})+\hat{\chi}(t) \\
\dot{\hat{\chi}} & =-K_{2} \Phi_{2}(\tilde{\sigma})
\end{aligned}
$$

where $\tilde{\sigma}(t)$ and $\tilde{\chi}(t)$ are respectively the estimation error of the ESO and the estimation error of the disturbance $\chi(t) . \hat{\sigma}(t)$ and $\hat{\chi}(t)$ are the observer 
internal states. $\dot{\hat{\sigma}}(t)$ and $\dot{\hat{\chi}}(t)$ are the dynamics of the observer internal states and the vectors $\Phi_{1}(\tilde{\sigma})=\left[\phi_{11}, \phi_{12}, \cdots, \phi_{16}\right]^{T}$ and $\Phi_{2}(\tilde{\sigma})=\left[\phi_{21}, \phi_{22}, \cdots, \phi_{26}\right]^{T}$ and each element of the mentioned vectors is given by:

$$
\begin{aligned}
& \phi_{1 i}\left(\tilde{\sigma}_{i}\right)=\mu_{1 i}\left|\tilde{\sigma}_{i}\right|^{1 / 2} \operatorname{sgn}\left(\tilde{\sigma}_{i}\right)+\mu_{2 i} \tilde{\sigma}_{i} \\
& \phi_{2 i}\left(\tilde{\sigma}_{i}\right)=\frac{1}{2} \mu_{1 i}^{2} \operatorname{sgn}\left(\tilde{\sigma}_{i}\right)+\frac{3}{2} \mu_{1 i} \mu_{2 i}\left|\tilde{\sigma}_{i}\right|^{1 / 2} \operatorname{sgn}\left(\tilde{\sigma}_{i}\right)+\mu_{2 i}^{2} \tilde{\sigma}_{i}
\end{aligned}
$$

where $\mu_{1 i}, \mu_{2 i} \geq 0$ with $i=\overline{1,6}, K_{1}=\operatorname{diag}\left(k_{11}, k_{12}, \cdots, k_{16}\right)$ and $K_{2}=$ $\operatorname{diag}\left(k_{21}, k_{22}, \cdots, k_{26}\right)$ are the observer gains which are definite positive matrices. Moreover, if each element of the observer gain matrices is selected as follows:

$$
\begin{aligned}
& \dot{k}_{1 i}(t)= \begin{cases}\omega_{i} \sqrt{\frac{\varsigma_{i}}{2}} & \text { if } \tilde{\sigma} \neq 0 \\
0 & \text { if } \tilde{\sigma}=0\end{cases} \\
& k_{2 i}(t)=2 \epsilon_{i} k_{1 i}(t)+\beta_{i}+4 \epsilon_{i}^{2}
\end{aligned}
$$

where $\omega_{i}, \varsigma_{i}, \beta_{i}$ and $\epsilon_{i}$ are arbitrary positive constants, with $i=\overline{1,6}$. Then, for any initial condition $\tilde{\sigma}_{i}(0)$ and $\tilde{\chi}_{i}(0)$, the variables $\tilde{\sigma}_{i}$ and $\tilde{\chi}_{i}$ will tend to zero in a finite time as is stated in the next.

Theorem 1. Consider the perturbed augmented system (13). The proposed AGSTA-ESO (14) ensures that the observer error dynamics converges to zero in finite time if the gains $K_{1}$ and $K_{2}$ are definite positive matrices according to the adaption law given by equations (15) and (16).

Proof. The observer error dynamics is given by:

$$
\begin{aligned}
& \dot{\tilde{\sigma}}=\tilde{\chi}(t)-K_{1} \Phi_{1}(\tilde{\sigma}) \\
& \dot{\tilde{\chi}}=-K_{2} \Phi_{2}(\tilde{\sigma})-h(t)
\end{aligned}
$$

Now, let us rename the error variables $\tilde{\sigma}, \tilde{\chi}$ as follows :

$$
\begin{aligned}
& s_{1 i}=\tilde{\sigma}_{i} \\
& s_{2 i}=\tilde{\chi}_{i}
\end{aligned}
$$

Then, Eq. (17) can be rewritten in a scalar form $(i=\overline{1,6})$ as:

$$
\begin{aligned}
& \dot{s}_{1 i}=-k_{1 i}\left[\mu_{1 i}\left|s_{1 i}\right|^{\frac{1}{2}} \operatorname{sgn}\left(s_{1 i}\right)+\mu_{2 i} s_{1 i}\right]+s_{2 i} \\
& \dot{s}_{2 i}=-k_{2 i}\left[\frac{1}{2} \mu_{1 i}^{2} \operatorname{sgn}\left(s_{1 i}\right)+\frac{3}{2} \mu_{1 i} \mu_{2 i}\left|s_{1 i}\right|^{\frac{1}{2}} \operatorname{sgn}\left(s_{1 i}\right)+\mu_{2 i}^{2} s_{1 i}\right]+h_{i}(t)
\end{aligned}
$$


Without loss of generality, we can represent the system (18) with the following simplified notation:

$$
\begin{aligned}
& \dot{s}_{1}=-k_{1}\left[\mu_{1}\left|s_{1}\right|^{\frac{1}{2}} \operatorname{sgn}\left(s_{1}\right)+\mu_{2} s_{1}\right]+s_{2} \\
& \dot{s}_{2}=-k_{2}\left[\frac{1}{2} \mu_{1}^{2} \operatorname{sgn}\left(s_{1}\right)+\frac{3}{2} \mu_{1} \mu_{2}\left|s_{1}\right|^{\frac{1}{2}} \operatorname{sgn}\left(s_{1}\right)+\mu_{2}^{2} s_{1}\right]+h(t)
\end{aligned}
$$

Now, in order to prove the asymptotic stability of the closed-loop system (19), we propose the following Lyapunov function candidate:

$$
V\left(s_{1}, s_{2}, k_{1}, k_{2}\right)=V_{0}(\cdot)+\frac{1}{2 \varsigma_{1}}\left(k_{1}-k_{1}^{*}\right)^{2}+\frac{1}{2 \varsigma_{2}}\left(k_{2}-k_{2}^{*}\right)^{2}
$$

where $\varsigma_{1}, \varsigma_{2}, k_{1}^{*}$, and $k_{2}^{*}$ are positive constants and $V_{0}(\cdot)$ is given by:

$$
V_{0}\left(s_{1}, s_{2}, k_{1}, k_{2}\right)=\xi^{T} P \xi
$$

with:

$$
\xi^{T}=\left[\phi_{1}\left(s_{1}\right), s_{2}\right]
$$

and

$$
P=P^{T}=\left[\begin{array}{cc}
\beta+4 \epsilon^{2} & -2 \epsilon \\
-2 \epsilon & 1
\end{array}\right]>0
$$

Since $\beta$ and $\epsilon$ are defined as arbitrary positive constants, $P$ is a positive definite matrix. Clearly, the quadratic form $V_{0}(\cdot)$ satisfies:

$$
\lambda_{\min }(P)\|\xi\|_{2}^{2} \leq V_{0}(s, k) \leq \lambda_{\max }(P)\|\xi\|_{2}^{2}
$$

where $\lambda_{\min }(P)$ and $\lambda_{\max }(P)$ respectively are the smallest and greatest eigenvalue of $P$. $\|\xi\|_{2}^{2}=\mu_{1}^{2}\left|s_{1}\right|+2 \mu_{1} \mu_{2}\left|s_{1}\right|^{\frac{3}{2}}+\mu_{2}^{2} s_{1}^{2}+s_{2}^{2}$ is the Euclidean norm of $\xi$ and the next inequality is satisfied as well:

$$
\left|\phi\left(s_{1}\right)\right| \leq\|\xi\|_{2} \leq \frac{V^{\frac{1}{2}}(\xi)}{\lambda_{\min }^{\frac{1}{2}}(P)}
$$

Finally, it is important to note that the proposed candidate Lyapunov function $V\left(s_{1}, s_{2}, k_{1}, k_{2}\right)$ is a continuous, positive definite and differentiable function. 
To compute the time derivative of the proposed Lyapunov function candidate (20), the time derivative of $V_{0}(\cdot)$ is found first. Then, the total time derivative of $V(\cdot)$ is computed:

Step 1. Noting that $\phi_{2}\left(s_{1}\right)=\phi_{1}^{\prime}\left(s_{1}\right) \phi_{1}\left(s_{1}\right)$, where $\phi_{1}^{\prime}\left(s_{1}\right)=\left(\mu_{1} \frac{1}{2\left|s_{1}\right|^{1 / 2}}+\right.$ $\left.\mu_{2}\right)$, and introducing $L=\frac{h(t)}{\phi_{1}^{\prime}\left(s_{1}\right)}$. Then, the time derivative of $V_{0}(\cdot)$ is obtained as:

$$
\begin{aligned}
& \dot{V}_{0}=2 \xi^{T} P \dot{\xi} \\
& =2 \xi^{T} P\left[\begin{array}{c}
\phi_{1}^{\prime}\left[-k_{1} \phi_{1}\left(s_{1}\right)+s_{2}\right] \\
-k_{2} \phi_{2}\left(s_{1}\right)+h(t)
\end{array}\right] \\
& =2 \xi^{T} P\left[\begin{array}{l}
\phi_{1}^{\prime}\left(s_{1}\right)\left[-k_{1} \phi_{1}\left(s_{1}\right)+s_{2}\right. \\
\phi_{1}^{\prime}\left(s_{1}\right) \phi_{1}\left(s_{1}\right)\left[-k_{2}+L\right]
\end{array}\right] \\
& =\phi_{1}^{\prime}\left(s_{1}\right) 2 \xi^{T} P \underbrace{\left[\begin{array}{cc}
-k_{1} & s_{2} \\
-\left(k_{2}-L\right) & 0
\end{array}\right]}_{A(t, \chi)} \xi \\
& =\phi_{1}^{\prime}\left(s_{1}\right) \xi^{T}\left(A^{T}(t, \chi) P+P A(t, \chi)\right) \xi \\
& =-\phi_{1}^{\prime}\left(s_{1}\right) \xi^{T} Q(t, \chi) \xi
\end{aligned}
$$

where

$$
Q(t, \chi)=\left[\begin{array}{cc}
2 k_{1}\left(\beta+4 \epsilon^{2}\right)-4 \epsilon\left(k_{2}-L\right) & \star \\
k_{2}-L-2 \epsilon k_{1}-\beta-4 \epsilon^{2} & 2 \epsilon
\end{array}\right]
$$

173 and $\star=k_{2}-L-2 \epsilon k_{1}-\beta-4 \epsilon^{2}$.

Selecting the gain $k_{2}=2 \epsilon k_{1}+\beta+4 \epsilon^{2}$, we have:

$$
Q-2 \epsilon I=\left[\begin{array}{cc}
2 k_{1} \beta-4 \epsilon\left(\beta+4 \epsilon^{2}-L\right)-2 \epsilon & -L \\
-L & 2 \epsilon
\end{array}\right]
$$

The matrix $\mathrm{Q}$ will be positive definite with a minimal eigenvalue $\lambda_{\min }(Q) \geq 2 \epsilon$ if

$$
k_{1}>\delta_{0}+\frac{\alpha_{2}^{2}}{4 \epsilon \beta}+\frac{\epsilon\left[2\left(\beta+4 \epsilon^{2}+L\right)+1\right]}{2 \beta}
$$

where $\delta_{0}$ is a small positive constant. 
Then, the time derivative of $V_{0}(\cdot)$ can be rewritten as:

$$
\dot{V}_{0}=-\phi_{1}^{\prime}\left(s_{1}\right) \xi^{T} Q(t, x) \xi \leq-2 \epsilon \phi_{1}^{\prime}\left(s_{1}\right) \xi^{T} \xi=-2 \epsilon\left(\mu_{1} \frac{1}{2\left|s_{1}\right|^{\frac{1}{2}}}+\mu_{2}\right) \xi^{T} \xi
$$

Finally, using Eq. (25), the time derivative of $V_{0}(\cdot)$ is expressed as:

$$
\begin{aligned}
\dot{V}_{0} & \leq-\frac{\epsilon \lambda_{\min }^{\frac{1}{2}}(P)}{\lambda_{\max }(P)} \mu_{1} V_{0}^{\frac{1}{2}}(s, k)-\frac{2 \epsilon}{\lambda_{\max }(P)} \mu_{2} V(s, k) \\
& \leq-\gamma V_{0}^{\frac{1}{2}}(s, k)
\end{aligned}
$$

175 with $\gamma=\mu_{1} \frac{\epsilon \lambda_{\min }^{\frac{1}{2}}(P)}{\lambda_{\max }(P)}$.

Step 2. The time derivate of the Lyapunov function candidate 20 is obtained as follows:

$$
\begin{aligned}
\dot{V} & =\dot{V}_{0}(\cdot)+\frac{1}{\varsigma_{1}}\left(k_{1}-k_{1}^{*}\right) \dot{k}_{1}+\frac{1}{\varsigma_{2}}\left(k_{2}-k_{2}^{*}\right) \dot{k}_{2} \\
& \leq-\gamma V_{0}^{\frac{1}{2}}(s, k)+\frac{1}{\varsigma_{1}}\left(k_{1}-k_{1}^{*}\right) \dot{k}_{1}+\frac{1}{\varsigma_{2}}\left(k_{2}-k_{2}^{*}\right) \dot{k}_{2} \\
& =-\gamma V_{0}^{\frac{1}{2}}(s, k)-\frac{\omega_{1}}{\sqrt{2 \varsigma_{1}}}\left|k_{1}-k_{1}^{*}\right|-\frac{\omega_{2}}{\sqrt{2 \varsigma_{2}}}\left|k_{2}-k_{2}^{*}\right|+\frac{1}{\varsigma_{1}}\left(k_{1}-k_{1}^{*}\right) \dot{k}_{1}+ \\
& +\frac{1}{\varsigma_{2}}\left(k_{2}-k_{2}^{*}\right) \dot{k}_{2}+\frac{\omega_{1}}{\sqrt{2 \varsigma_{1}}}\left|k_{1}-k_{1}^{*}\right|+\frac{\omega_{2}}{\sqrt{2 \varsigma_{2}}}\left|k_{2}-k_{2}^{*}\right|
\end{aligned}
$$

Using the inequality $\sqrt{x^{2}+y^{2}+z^{2}} \leq|x|+|y|+|z|$, the first three terms of $\dot{V}$ can be synthesized as follows:

$$
-\gamma V_{0}^{\frac{1}{2}}(s, k)-\frac{\omega_{1}}{\sqrt{2 \varsigma_{1}}}\left|k_{1}-k_{1}^{*}\right|-\frac{\omega_{2}}{\sqrt{2 \varsigma_{2}}}\left|k_{2}-k_{2}^{*}\right| \leq-\pi \sqrt{V\left(s, k_{1}, k_{2}\right)}
$$

176

where $\pi=\min \left(\gamma, \omega_{1}, \omega_{2}\right)$.

Assuming that there exist positive constants $k_{1}^{*}$ and $k_{2}^{*}$ such that $k_{1}-k_{1}^{*}<$ 0 and $k_{2}-k_{2}^{*}<0$ are satisfied $\forall t \geq 0$. Then, the time derivative of $V$ can be rewritten as:

$$
\begin{aligned}
\dot{V} & \leq-\pi \sqrt{V\left(s, k_{1}, k_{2}\right)}-\left|k_{1}-k_{1}^{*}\right|\left(\frac{1}{\varsigma_{1}} \dot{k}_{1}-\frac{\omega_{1}}{\sqrt{2 \varsigma_{1}}}\right)-\left|k_{2}-k_{2}^{*}\right|\left(\frac{1}{\varsigma_{2}} \dot{k}_{2}-\frac{\omega_{2}}{\sqrt{2 \varsigma_{2}}}\right) \\
& =-\pi \sqrt{V\left(s, k_{1}, k_{2}\right)}+\vartheta
\end{aligned}
$$


where:

$$
\vartheta=-\left|k_{1}-k_{1}^{*}\right|\left(\frac{1}{\varsigma_{1}} \dot{k}_{1}-\frac{\omega_{1}}{\sqrt{2 \varsigma_{1}}}\right)-\left|k_{2}-k_{2}^{*}\right|\left(\frac{1}{\varsigma_{2}} \dot{k}_{2}-\frac{\omega_{2}}{\sqrt{2 \varsigma_{2}}}\right)
$$

In order to preserve the finite time convergence, it is necessary to ensure the condition $\vartheta=0$, which will be achieved through the following adaption laws:

$$
\begin{aligned}
& \dot{k}_{1}=\omega_{1} \sqrt{\frac{\varsigma_{1}}{2}} \\
& \dot{k}_{2}=\omega_{2} \sqrt{\frac{\varsigma_{2}}{2}}
\end{aligned}
$$

In brief, the adaptive gains $k_{1}$ and $k_{2}$ will be increased based on the dynamic and algebraic equations stated in (15)-(16), until the condition (34) is reached. Then, the matrix $Q$ will be positive definite and the finite time convergence will be assured according to (43). The previous result guarantees the finite time convergence of $\tilde{\sigma}$ and $\tilde{\chi}$ to zero, and when this happens, the adaptive gains $k_{1}$ and $k_{2}$ will stop growing by making $\dot{k}_{1}=0$.

Remark 2. In real applications, an 'ideal' sliding mode cannot be established [30]. Then, it is crucial to introduce the concept of the 'real' sliding mode. Definition 1. Given the sliding variable $\tilde{\sigma}(t)$, the 'real sliding surface' associated with (9) is defined as in the sense of [31].

In this context, the definition of 'real' sliding mode means that the condition $\tilde{\sigma}(t)=0$ is never satisfied. In fact, the variable $\tilde{\sigma}(t)$ is related to the system state $\eta$ as stated by Eq. (11). Then, the measurement of $\eta$ is always corrupted by noise, which makes impossible the satisfaction of the condition $\tilde{\sigma}(t)=0$. Therefore, the gains $k_{1}(t)$ and $k_{2}(t)$ given by Eqs. (15) and (16) will increase drastically. To overcome this drawback, the definition of the real sliding surface is employed, and the adaptive gains (15)-(16) are modified as follows:

$$
\begin{aligned}
& \dot{k}_{1 i}(t)= \begin{cases}0 & \text { if }|\tilde{\sigma}|<\delta_{i} \\
\mu_{1 i} \sqrt{\frac{\varsigma_{1 i}}{2}} & \text { otherwise }\end{cases} \\
& k_{2 i}(t)=2 \epsilon_{i} k_{1 i}(t)+\beta_{i}+4 \epsilon_{i}^{2}
\end{aligned}
$$

where $\delta_{i}$ is a small positive parameter. 
Remark 3. The selection of the parameter $\delta_{i}$ was chosen by trial and error approach. However, there is a trade-off between the parameter value selection and the chattering effect in the robot's actuators caused by the gain overestimation. This means that if $\delta_{i}$ is selected close to zero, the feedback controller's gains will grow up, and if the the parameter is selected too high, the convergence time of the robot to the reference trajectory will increase as well.

\section{Enhanced Controller design}

In this section, a brief description of the enhanced backstepping and nonlinear PD controllers is provided. First, The adaptive disturbance observer is introduced into the controllers in order to counteract the effects of the external disturbances and parameter uncertainties. Then, Lyapunov's functions are used to prove the stability of the controller/observer scheme.

\subsection{Adaptive Backstepping controller}

The design of a Backstepping controller for trajectory tracking in-depth and yaw dynamics was proposed in work [14. In this study, the authors demonstrated the effectiveness of the proposed controller through real-time experiments. However, from the depth tracking experimental results, one can notice that there is a tracking offset between the controller signal and the trajectory reference. This deficiency can be due to a bad tuning or that the force of the disturbance is excessive and, therefore, exceeds the capabilities of the proposed control. In order to overcome this drawback, in this section, the disturbance estimation made by the AGSTA-ESO is inserted into the algorithm shown in [14. As a result of the proposed scheme, the Adaptive Backstepping control (ABS) is described in the following theorem:

Theorem 2. Consider the system (7) transformed into (9). Let $e_{1}(t)=\zeta_{1}-$ $\zeta_{1}^{d}$ and $e_{2}(t)=\left[e_{2}^{x}(t), e_{2}^{y}(t), \cdots, e_{2}^{\psi}(t)\right]^{T}=\dot{e}_{1}(t)+\Gamma e_{1}(t)$ be the tracking errors and the gain diagonal and positive definite matrices $\Gamma=\operatorname{diag}\left(\gamma_{1}, \gamma_{2}, \cdots, \gamma_{6}\right)$ and $\Upsilon=\operatorname{diag}\left(v_{1}, v_{2}, \cdots, v_{6}\right)$. If Assumptions 1 and 2 are satisfied and proposing the following adaptive backstepping controller:

$$
\tau_{\eta}=G(\zeta)^{-1}\left[\ddot{\eta}^{d}-e_{1}-\bar{F}(\zeta)+\Gamma\left(\Gamma e_{1}-e_{2}\right)-\Upsilon e_{2}-\hat{d}-\hat{K} \operatorname{Sign}\left(e_{2}\right)\right]
$$


where $\hat{d}(t)$ is the estimation of the disturbance made by the AGSTA-ESO (14), $\operatorname{Sign}\left(e_{2}(t)\right)=\left[\operatorname{sgn}\left(e_{2}^{x}(t)\right), \operatorname{sgn}\left(e_{2}^{y}(t)\right), \cdots, \operatorname{sgn}\left(e_{2}^{\psi}(t)\right)\right]^{T}$, and $\hat{K}$ is a constant gain selected by the following adaption law:

$$
\dot{\hat{K}}=\lambda_{1}\left\|e_{2}(t)\right\|
$$

Proof. Step 1. Let us consider the following Lyapunov function candidate:

$$
V_{1}\left(e_{1}\right)=\frac{1}{2} e_{1}^{T} e_{1}
$$

The time derivative of $V$ along the trajectories of the system $(9)$ is given by:

$$
\dot{V}\left(e_{1}\right)=e_{1}^{T} \dot{e}_{1}=e_{1}^{T}\left(\dot{\zeta}_{1}-\dot{\zeta}_{1}^{d}\right)=e_{1}^{T}\left(\zeta_{2}-\dot{\zeta}_{1}^{d}\right)
$$

Selecting $\zeta_{2}$ as the virtual control, $\zeta_{2}^{v}$, as follows:

$$
\zeta_{2}^{v}=\dot{\zeta}_{1}^{d}-\Gamma e_{1}
$$

yields to:

$$
\dot{V}_{1}\left(e_{1}\right)=-e_{1}^{T} \Gamma e_{1}
$$

Moreover, the stabilization of $\dot{e}_{1}(t)$ is achieved by choosing $\Gamma>0$.

Step 2. Introducing the following error $e_{2}=\zeta_{2}-\zeta_{2}^{v}$, and computing its time derivative with (53), yields to the following dynamic error system:

$$
\begin{aligned}
& \dot{e_{1}}=e_{2}-\Gamma e_{1} \\
& \dot{e_{2}}=\bar{F}(\zeta)+G(\zeta) u+d(t)-\ddot{\zeta}_{1}^{d}+\Gamma \dot{e}_{1}
\end{aligned}
$$

Proposing the Lyapunov function candidate as:

$$
V_{2}\left(e_{1}, e_{2}\right)=\frac{1}{2} e_{1}^{T} e_{2}+\frac{1}{2} e_{2}^{T} e_{2}+\frac{1}{2 \lambda_{1}} \tilde{K}^{2}
$$

221 where the parameter estimation error is defined as $\tilde{K}=\hat{K}-K$. 
The time derivative of $V_{2}$ along the trajectories over the system (55) is obtained as follows:

$$
\begin{aligned}
\dot{V}_{2} & =e_{1}^{T} \dot{e}_{1}+e_{2}^{T} \dot{e}_{2}+\frac{1}{\lambda_{1}} \tilde{K} \dot{\hat{K}} \\
& =e_{1}^{T}\left[e_{1}-\Gamma e_{1}\right]+e_{2}^{T}\left[\bar{F}(\zeta)+G(\zeta) \tau_{\eta}+d(t)-\ddot{\zeta}_{1}^{d}+\Gamma \dot{e}_{1}\right]+\frac{1}{\lambda_{1}} \tilde{K} \dot{\hat{K}} \\
& =-e_{1}^{T} \Gamma e_{1}+e_{2}^{T}\left[e_{1}+\bar{F}(\zeta)+G(\zeta) \tau_{\eta}+d(t)-\ddot{\zeta}_{1}^{d}+\Gamma \dot{e}_{1}\right]+\frac{1}{\lambda_{1}} \tilde{K} \dot{\hat{K}}
\end{aligned}
$$

Injecting the control law 49 into the time derivative of $V_{2}$, and assuming that the AGSTA-ESO estimates the external disturbance in finite time and fulfills the following constraint $\|d(t)-\hat{d}(t)\| \leq K$ [32], leads to

$$
\begin{aligned}
\dot{V}_{2} & =-e_{1}^{T} \Gamma e_{1}-e_{2}^{T} \Upsilon e_{2}+e_{2}^{T}\left[d(t)-\hat{d}(t)-\hat{K} \operatorname{Sgn}\left(e_{2}\right)\right]+\frac{1}{\lambda_{1}} \tilde{K} \dot{\hat{K}} \\
& \leq-\lambda_{\min }(\Gamma)\left\|e_{1}\right\|_{2}^{2}-\lambda_{\min }(\Upsilon)\left\|e_{2}\right\|_{2}^{2}+\left\|e_{2}\right\|_{2} K-\left\|e_{2}\right\|_{2} \hat{K}+\tilde{K}\left\|e_{2}\right\|_{2} \\
& =-\lambda_{\min }(\Gamma)\left\|e_{1}\right\|_{2}^{2}-\lambda_{\min }(\Upsilon)\left\|e_{2}\right\|_{2}^{2}
\end{aligned}
$$

\subsection{Adaptive Nonlinear PD control}

The NLPD is a robust controller proposed in work [18]. The authors have proven the robustness of the NLPD to parametric uncertainties. However, based on the experimental results, due to the extra damping added to the vehicle, the performance of the depth tracking shows a constant error during the steady-state behavior. In order to overcome this drawback, it was shown in Guerrero et al. [29] that, the addition of a finite-time disturbance estimator, GSTA-ESO, to the nominal NLPD improves significantly its effectiveness towards external and parametric disturbances. In this vein, one step ahead might be the introduction of adaptation laws allowing to update the observer's gains depending on the disturbance evolution. The advantage is that the controller will be able of rejecting bounded time-varying disturbances even if the upper bound of the perturbation is not known. Following 
this line of thinking, the disturbance estimation made by the AGSTA-ESO will be introduced into the NLPD.

For notation consistency, let us now write the UAV's dynamic system (9) in terms of the variables $\zeta_{1}=\eta$ and $\zeta_{2}=\dot{\eta}$, namely,

$$
\hat{M}_{\zeta}(\zeta) \dot{\zeta}_{2}+\hat{C}_{\zeta}\left(\nu, \zeta_{1}\right) \dot{\zeta}_{1}+\hat{D}_{\zeta}\left(\nu, \zeta_{1}\right) \dot{\zeta}_{1}+\hat{g}_{\zeta}\left(\zeta_{1}\right)=\tau_{\zeta}+\bar{d}(t)
$$

The following Theorem gives the main result of this Section:

Theorem 3. Let the vehicle's mathematical model with external disturbances be defined by Eq. (7), which can be rewritten into the model (65). Introducing the disturbance estimation $\hat{d}(t)$ given by equations (14) into the following adaptive nonlinear PD (ANLPD) controller

$$
\begin{aligned}
\tau_{\zeta}=\hat{M}_{\zeta} \dot{\zeta}_{2}^{d}(t)+\hat{C}_{\zeta}(\nu, \zeta) \dot{\zeta}_{1}^{d}(t)+\hat{D}_{\zeta}(\nu, \eta) \dot{\zeta}_{1}^{d}(t)+\hat{g}_{\zeta}(\zeta)- & (66) \\
& -K_{p}(\cdot) e-K_{d}(\cdot) \dot{e}-\hat{M}_{\eta}(\eta) \hat{d}-\hat{K} \operatorname{Sign}(\dot{e})
\end{aligned}
$$

where $\dot{\zeta}_{2}^{d}(t)=\ddot{\zeta}_{1}^{d}(t), e(t)=\left[e_{1}(t), e_{2}(t), \cdots, e_{6}(t)\right]^{T}=\zeta_{1}(t)-\zeta_{1}^{d}(t)$ is the error signal, $\dot{e}(t)=\left[\dot{e}_{1}(t), \dot{e}_{2}(t), \cdots, \dot{e}_{6}(t)\right]^{T}=\dot{\zeta}_{1}(t)-\dot{\zeta}_{1}^{d}(t)$ its time derivative, and the desired trajectory is defined as $\zeta_{1}^{d}(t)=\left[x_{d}(t), y_{d}(t), z_{d}(t), \phi_{d}(t), \theta_{d}(t), \psi_{d}(t)\right]^{T}$. The vector $\operatorname{Sign}(\dot{e})=\left[\operatorname{sgn}\left(\dot{e}_{1}(t)\right), \operatorname{sgn}\left(\dot{e}_{2}(t)\right), \cdots, \operatorname{sgn}\left(\dot{e}_{6}(t)\right)\right], d(t)=\hat{M}(\eta)^{-1} \bar{d}(t)$, and $\hat{K}$ is a constant gain selected by the following adaption law:

$$
\dot{\hat{K}}=\lambda_{2}\left\|e_{2}(t)\right\|
$$

where $\lambda_{2}$ is a positive constant. The gain matrices $K_{p}(\cdot)$ and $K_{d}(\cdot)$ have the following structure:

$$
\begin{aligned}
K_{p}(\cdot)= & {\left[\begin{array}{cccc}
k_{p 1}(\cdot) & 0 & \cdots & 0 \\
0 & k_{p 2}(\cdot) & \cdots & 0 \\
\vdots & \vdots & \ddots & \vdots \\
0 & 0 & \cdots & k_{p n}(\cdot)
\end{array}\right]>0 } \\
K_{d}(\cdot)= & {\left[\begin{array}{cccc}
k_{d 1}(\cdot) & 0 & \cdots & 0 \\
0 & k_{d 2}(\cdot) & \cdots & 0 \\
\vdots & \vdots & \ddots & \vdots \\
0 & 0 & \cdots & k_{d n}(\cdot)
\end{array}\right]>0 }
\end{aligned}
$$


and asymptotically stabilize the system (7) if the underwater vehicle is moving at low speed, and if $k_{p j}(\cdot)$ and $k_{d j}(\cdot)$ are defined as:

$$
\begin{aligned}
k_{p j}(\cdot) & = \begin{cases}b_{p j}\left|e_{j}(t)\right|^{\left(\mu_{p j}-1\right)} & \text { if }\left|e_{j}(t)\right|>d_{p j} \\
b_{p j} d_{p j}^{\left(\mu_{p j}-1\right)} & \text { if }\left|e_{j}(t)\right| \leq d_{p j}\end{cases} \\
k_{d j}(\cdot) & = \begin{cases}b_{d j}\left|\dot{e}_{j}(t)\right|^{\left(\mu_{d j}-1\right)} & \text { if }\left|\dot{e}_{j}(t)\right|>d_{d j} \\
b_{d j} d_{d j}^{\left(\mu_{d j}-1\right)} & \text { if }\left|\dot{e}_{j}(t)\right| \leq d_{d j}\end{cases} \\
\forall \mu_{p j}, \mu_{d j} \in[0,1] . &
\end{aligned}
$$

227

and $\kappa$ is a positive constant.

This function is positive definite and radially unbounded (see [18] for a deeper description). Then, the time derivative of the Lyapunov candidate function is computed as:

$$
\dot{V}(e, \dot{e})=\dot{e}^{T} \hat{M}_{\zeta}(\zeta) \ddot{e}+\frac{1}{2} \dot{e}^{T} \dot{\hat{M}}_{\zeta}(\zeta) \dot{e}+e^{T} K_{p}(\cdot) \dot{e}+\frac{1}{\lambda_{2}} \tilde{K} \dot{\hat{K}}
$$

Considering that the GSTA-ESO converges to the disturbance dynamics in finite time, it is reasonable to assume that $\|d(t)-\hat{d}(t)\| \leq K$, with the unknown constant $K>0$. The constant $K$ was obtained through the adaption 
law (67). Then, substituting the error dynamics 72 into the time derivative of $V(\cdot)$ and considering the Assumption 2 and equation (67), yields to:

$$
\begin{aligned}
\dot{V}(e, \dot{e}) & =-\dot{e}^{T}\left[\hat{D}_{\zeta}(\nu, \zeta)+K_{d}(\cdot)\right] \dot{e}+\dot{e}^{T}[d(t)-\hat{d}(t)]-\hat{K} \dot{e}^{T} \operatorname{Sign}(\dot{e})+\frac{1}{\lambda_{2}} \tilde{K} \dot{\hat{K}} \\
& =-\dot{e}^{T}\left[\hat{D}_{\zeta}(\nu, \zeta)+K_{d}(\cdot)\right] \dot{e}+\dot{e}^{T}[d(t)-\hat{d}(t)]-\hat{K} \sum_{i=0}^{6}\left|\dot{e}_{i}\right|+\frac{1}{\lambda_{2}} \tilde{K} \dot{\hat{K}} \\
& \leq-\lambda_{\min }\left(\hat{D}_{\zeta}(\nu, \zeta)+K_{d}(\cdot)\right)\|\dot{e}\|^{2}+K\|\dot{e}\|-\hat{K}\|\dot{e}\|+\tilde{K}\|\dot{e}\| \\
& \leq-\lambda_{\min }\left(\hat{D}_{\zeta}(\nu, \zeta)+K_{d}(\cdot)\right)\|\dot{e}\|^{2}
\end{aligned}
$$

Finally, because the gain matrix is $K_{d}(\cdot)>0$ by design and the damping matrix fulfills $\hat{D}_{\zeta}(\nu, \zeta)>0$ [4], the function $\dot{V}(\cdot)$ is negative semi-definite. Finally, applying the Krasovskii-Lasalle's theorem we can conclude that the equilibrium point is asymptotically stable [18].

Remark 5. In the experimental part of this work, the performance of the developed controller law given by equation (66) is compared to the NLPD control proposed in [18], namely:

$$
\tau_{\zeta}=\hat{M}_{\zeta} \dot{\zeta}_{2}^{d}(t)+\hat{C}_{\zeta}(\nu, \zeta) \dot{\zeta}_{1}^{d}(t)+\hat{D}_{\zeta}(\nu, \eta) \dot{\zeta}_{1}^{d}(t)+\hat{g}_{\zeta}(\zeta)-K_{p}(\cdot) e-K_{d}(\cdot) \dot{e}
$$

Remark 6. It is worth to notice that in this manuscript is different from the work presented in [19]. On one hand, in study [19], a nonlinear PID was proposed while in this work, we propose the improvement of the nonlinear PD proposed by [18]. On the other hand, in this manuscript, we use sliding mode theory to improve a PD controller while in work [19] is an extension of the saturated based-control.

\section{Experimental Results}

Leonard is a tethered underwater vehicle entirely designed and built at LIRMM (University of Montpellier / CNRS, France). The vehicle's size is 75 $\mathrm{cm}$ long, $55 \mathrm{~cm}$ width, and $45 \mathrm{~cm}$ height and weighs $28 \mathrm{~kg}$. The propulsion system of this vehicle consists of six independent thrusters to obtain a fully actuated system. 
The information from the sensors of the underwater vehicle (depth, IMU) is sent, through a tether, to a computer located at the surface. Then, the machine computes the control laws and sends the control input to each thruster of Leonard. The computer machine is a laptop with Intel Core i7-3520M 2.9 $\mathrm{GHz} \mathrm{CPU}, 8 \mathrm{~GB}$ of RAM, it runs under Windows 7 operating system, and the control software is developed using Visual $\mathrm{C}++2010$. More details about the Leonard Underwater Vehicle can be found in [22].

The real-time experiments have been carried out in the $4 \times 4 \times 1.2 \mathrm{~m}$ pool of the LIRMM. Even though the proposed control laws were given by equations (49), 64), 66) and (80) is designed for the whole system (six degrees of freedom), the experiments conducted in this study concern only depth and yaw trajectory tracking. The primary goal of the proposed controllers is to track the desired reference trajectory robustly in depth and yaw even in the presence of parameter uncertainties and external disturbances. The whole set of real-time experiments is available at:

https://www.youtube.com/watch?v=aBOvvlsYNQE
https://www.youtube.com/watch?v=oXPqSLvXobk

\subsection{Proposed Scenarios}

In order to show the improvement of adding the adaptive finite time disturbance estimator to the BS and NLPD controllers, we propose three main scenarios:

(i) Scenario 1: Nominal case.

In this scenario, the robot follows a predefined desired trajectory in depth and yaw in the absence of external disturbances. During this test, the controller's gains are adjusted to obtain the best tracking. These gains remain unchanged during the rest of the experiments.

(ii) Scenario 2: Robustness towards parametric uncertainties

In this test, the buoyancy and damping of the vehicle are increased to test the robustness of the proposed methodology towards parametric uncertainties, see Figure 2 .

(iii) Scenario 3: External disturbances rejection.

In this test, the vehicle has the task of loading an object and when reaching a certain depth, dropping that object. Moreover, during this test, it is possible to see a sudden change in the vehicle's weight and how it affects the controller performance, see Figure 3. 


\subsection{Feedback controller gains tuning}

It is worth to notice that the selection of the tuning gains of the BS and NLPD controllers were found following the procedure proposed in [14] and [18, respectively.

For the tuning of the proposed adaptive observer gains given by Eq. (15), we found the gains by heuristic approach, the steps are shown below:

1. We set the value of $\varsigma_{1 i}=1$ and $\mu_{1 i}=0.1$. Note that $\mu_{1 i}$ is the main parameter to modify the value of $k_{1 i}$.

2. The gain $k_{2 i}$ is related to the estimation of the disturbance. In this vein, we set $\epsilon_{i}$ to a small value $\left(\epsilon_{i}=0.1\right)$ in order to limit the growth of $k_{2 i}$. Also, we use $\beta_{i}$ to modify the value of $k_{2 i}$.

3. It is worth to note that the ideal sliding surface does not exist, then, $\tilde{\sigma}=$ 0 is never reached and $\delta_{i}$ is a nonzero constant. Initially, the value of $\delta_{i}$ is set to a small value. This parameter needs to be adjusted depending of the behavior of the robot during experiments. For instance, if the gains increase fast, this value needs to be increased as well.

4. Finally, the parameter $\Lambda_{i}$ is used to modify the convergence rate of the robot to the reference trajectory. As explained above, this value can be set to $\Lambda=1$ and it will be increased to improve the controller performance.

Remark 7. The adaption law of $\hat{K}$ is obtained through the integration of Equations (50) or (67). One can notice that the adaption depends on the norm of the time derivative of the tracking error and the gains $\lambda_{1}$ or $\lambda_{2}$. Then, in order to minimize the chattering effect due to the signum function into the control law, it is suggested to keep the gains $\lambda_{1}$ and $\lambda_{2}$ as small as possible. In the real-time experiments, the gains were considered as $\lambda_{1} \rightarrow 0$ and $\lambda_{2} \rightarrow 0$, which yields to $\hat{K} \rightarrow 0$. The Tables 1 .4 show the gains for the proposed observer and controllers.

Remark 8. Note that at first sight, the proposed controller have several gains to tune, however, most of the parameters can be set to zero or to a small value. Based on the theoretical results of the main theorem, if the gains are selected as in Eq. 15, the robot will converge to the reference trajectory. 


\begin{tabular}{ccc}
\hline Depth & $\gamma_{3}=3.0$ & $v_{3}=1.9$ \\
Yaw & $\gamma_{6}=16.34$ & $v_{6}=36.65$ \\
\hline
\end{tabular}

Table 1: BS control gains used in real-time experiments

\begin{tabular}{cccc}
\hline Depth & $\mu_{3}=0.1$ & $\varsigma_{3}=1.0$ & $\Lambda_{3}=2.0$ \\
& $\epsilon_{3}=0.1$ & $\beta_{3}=0.33$ & $\delta_{3}=0.01$ \\
Yaw & $\mu_{6}=0.1$ & $\varsigma_{6}=1.0$ & $\Lambda_{6}=3.5$ \\
& $\epsilon_{6}=0.1$ & $\beta_{6}=0.01$ & $\delta_{6}=0.2$ \\
\hline
\end{tabular}

Table 2: Adaptive disturbance observer gains for the BS scheme

313

\subsection{Tracking Performance Indexes}

In order to evaluate the tracking performance of the proposed controllers, let us compute the Root Mean Square Error (RMSE) as follows:

$$
R M S(\cdot(t))=\sqrt{\frac{1}{T_{f}} \int_{0}^{T_{f}}|\cdot(t)|^{2} d t}
$$

In addition, the integral of control inputs (the applied force and torque) are computed to estimated the energy consumption used in each case, that is:

$$
I N T=\int_{t_{1}}^{t_{2}}|\tau(t)| d t
$$

where $t_{1}=3 \mathrm{~s}$ and $t_{2}=50 \mathrm{~s}$.

In the rest of the paper, the RMSE for yaw and depth are defined as $R M S E_{\psi}, R M S E_{z}$, respectively. The expressions $I N T_{\psi}$ and $I N T_{z}$ are the integral control input of yaw and depth, respectively.

The estimated values for the integral are listed in Tables 7.8 .

\subsection{Scenario 1: Nominal Case}

In this test, the submarine Leonard follows a predefined trajectory in depth and heading at the same time. For the depth test, the robot goes from the surface to a depth of $30 \mathrm{~cm}$. At this point, the vehicle remains stable for 20 seconds, and then, it goes to $20 \mathrm{~cm}$, where it remains until the end of the test. For the heading task, the vehicle turns from its initial position to 60 degrees, where it remains stable for 20 seconds. Then, the submarine 


\begin{tabular}{cccc}
\hline Depth & $b_{p 3}=20$ & $d_{p 3}=0.05$ & $\mu_{p 3}=0.1$ \\
& $b_{p 3}=13$ & $d_{p 3}=0.25$ & $\mu_{p 3}=0.2$ \\
Yaw & $b_{p 3}=4.5$ & $d_{p 3}=0.015$ & $\mu_{p 3}=0.2$ \\
& $b_{p 3}=0.2$ & $d_{p 3}=0.15$ & $\mu_{p 3}=0.2$ \\
\hline
\end{tabular}

Table 3: NLPD control gains used in real-time experiments

\begin{tabular}{cccc}
\hline Depth & $\mu_{3}=0.1$ & $\varsigma_{3}=1.0$ & $\Lambda_{3}=2.0$ \\
& $\epsilon_{3}=0.1$ & $\beta_{3}=0.33$ & $\delta_{3}=0.01$ \\
Yaw & $\mu_{6}=0.1$ & $\varsigma_{6}=1.0$ & $\Lambda_{6}=2.0$ \\
& $\epsilon_{6}=0.1$ & $\beta_{6}=0.01$ & $\delta_{6}=0.2$ \\
\hline
\end{tabular}

Table 4: Adaptive disturbance observer gains for the NLPD scheme

turns to -60 degrees in only 6 seconds, where the vehicle stays until the end of the trial. The top of Fig. 4 shows the trajectory tracking in depth and yaw for the nominal case. From the Fig. 4, one can notice that all the controllers have a good tracking performance, and this can be confirmed through the plot of the tracking errors, which is shown in the middle of the Fig. 4 and is numerically expressed for the RMSE measurement in Tables 5 and 6. In fact, from the RMSE indicator is easy to observe the improvement when the adaptive observer is considered. Then, at the bottom of Fig. 4, the evolution of the control inputs is shown. Again, the behavior of all the proposed controllers remains similar.

The top of figures 6 and 8 show the evolution of the adaptive gains of the proposed disturbance observer. From these figures, we can observe that when the vehicle moves from the steady position to another point, the gains are automatically adjusted in order to minimize the tracking error.

In the top of figs. 9 10, the estimation made by the disturbance observer is shown. The estimation of the disturbance using the Backstepping controller is displayed in the upper part of Fig. 9, while the estimation employing the NLPD is shown at the top of Fig. 10. It is worth to note that both estimations provided by the observers are not precisely the same, this is reasonable because the controllers are different and do not have the same gains. Nevertheless, the shape of the estimated disturbance is similar for both cases.

Finally, to compute the energy consumption of each controller and to 
establish a fair comparison, the nominal controller is compared against its improved version. For example, for the Backstepping controller case:

$$
I N T_{z}=\frac{I N T_{z}(A B S)}{I N T_{z}(B S)}=1.02 ; I N T_{\psi}=\frac{I N T_{\psi}(B S)}{I N T_{\psi}(A B S)}=1.18
$$

This result means that the energy consumption for the trajectory tracking in depth is almost similar for both controllers. While the energy consumption for the tracking in heading for the nominal BS is 1.18 time higher than the energy consumption using the ABS. The following ratios gives the integral of control input indicator for the NLPD controller:

$$
I N T_{z}=\frac{I N T_{z}(N L P D)}{I N T_{z}(A N L P D)}=1.02 ; I N T_{\psi}=\frac{I N T_{\psi}(A N L P D)}{I N T_{\psi}(N L P D)}=1.16
$$

The energy consumption for the tracking test in depth is similar for both controllers. For the trajectory tracking in yaw, the consumption of the ANLPD controller is 1.16 time higher than the energy consumption using the nominal design.

\subsection{Scenario 2: Robustness to parametric uncertainties}

In this scenario, to demonstrate the robustness of the proposed adaptive disturbance observer, the physical parameters of Leonard have been modified. Firstly, two floaters have been attached to both sides of the submarine to increase the buoyancy of the vehicle by $+100 \%$. Secondly, to increase the rotational damping along $\mathrm{z}$ by approximately 90\%, a large rigid sheet of plastic that has a dimension of $45 \times 10 \mathrm{~cm}$ has been attached on one side of the submarine (see Fig. 2).

In the top of Fig. 5, one can observe the behavior of the proposed controllers. From the Figure, it is possible to note that the nominal design of the BS and NLPD shows an offset between the trajectory of the submarine and the reference signal. The results shown in this work coincide with the ones presented in [14] and [18]. In contrast with these previous results, the introduction of the adaptive observer allows improving the performance of the nominal control design by suppressing the signal offset. The improved controllers are capable of following the reference trajectory despite the uncertainties in the parameters. The plot of the tracking errors is shown in the middle of Fig. 5 and the numerical expression of the errors are shown in Tables 5 and 6. The evolution of the input signals is displayed at the bottom 
of Fig. 5. As expected, at the beginning of the test, the improved version of the controllers demands more energy than the nominal designs, but once the reference is reached, the behaviors are similar for both schemes. The values of the integral of the control inputs for the tracking test using the BS and ABS control shows that the energy consumption is similar for both cases, and the following ratios also illustrate this:

$$
I N T_{z}=\frac{I N T_{z}(A B S)}{I N T_{z}(B S)}=1.01 ; I N T_{\psi}=\frac{I N T_{\psi}(A B S)}{I N T_{\psi}(B S)}=1.0
$$

For the case of the depth and yaw tracking using the NLPD and ANLPD, the ratios are given by

$$
I N T_{z}=\frac{I N T_{z}(N L P D)}{I N T_{z}(A N L P D)}=1.04 ; I N T_{\psi}=\frac{I N T_{\psi}(A N L P D)}{I N T_{\psi}(N L P D)}=1.02
$$

This means that the energy consumption of the NLPD control is only 1.04 times higher than the consumption of the ANLPD. Moreover, the energy consumption for the heading tracking is also very similar for both methodologies.

The adaptive gains of the proposed observer for the ABS and ANLPD controllers are shown in the middle of the Figures 6 and 8 , respectively. The estimated disturbance for the ABS is displayed at the middle of the Fig. 10 . while the estimation made by the ANLPD is shown at the middle of the Fig. 10. Again, the estimated disturbance shape is similar for both methods.

\subsection{Scenario 3:Rejection of external disturbances}

This test is inspired by a more realistic situation where the submarine is equipped with a robotic manipulator. The main objective is to transport an object from a certain depth to another where the submarine will release the load. In this context, to simulate an object, we tie a metallic $1 \mathrm{~kg}$ block to Leonard with a $20 \mathrm{~cm}$-long line. In this test, the maximal depth has been set to $40 \mathrm{~cm}$. As the maximum depth of the basin is $50 \mathrm{~cm}$, the robot will be suddenly disturbed when it will reach $30 \mathrm{~cm}$, because the metallic block will touch the floor, thus suddenly canceling its weight's effect. The disturbance will be acting on the robot until it starts to move up and reaches $30 \mathrm{~cm}$. Then, the action of the extra weight will influence the trajectory of the submarine again (see Fig. 3). This action simulates both the sudden release and recovery of a load by the robot. 


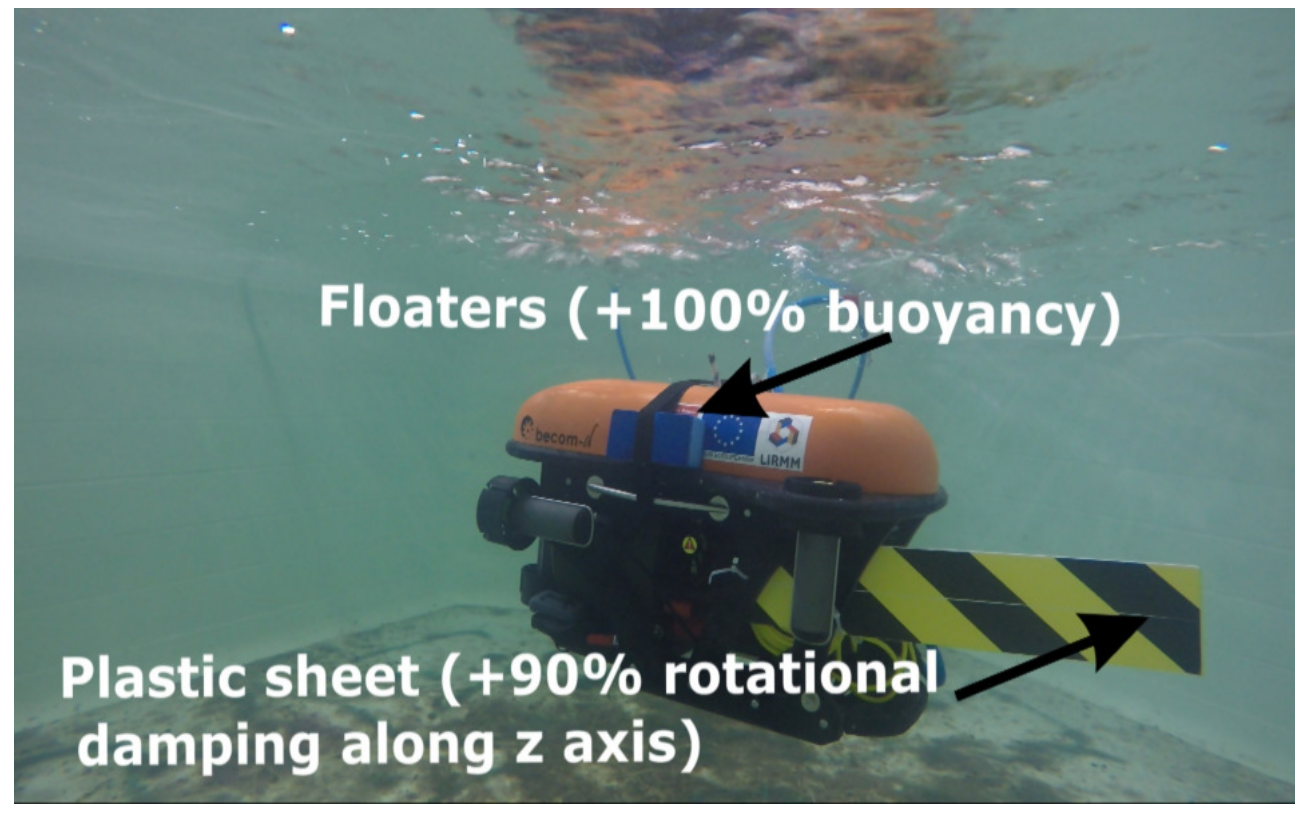

Figure 2: Modification of system parameters, increasing the buoyancy force and damping along $\mathrm{z}$ axis.

The performance of the proposed controllers is shown at the top of Fig. 7. From this figure, it can be noticed that although the behavior of the BS and the ABS is similar at the beginning of the test, the BS fails to track the reference signal efficiently when the robot tries to reach the 20 centimeters in depth. Moreover, the BS control has a $10 \mathrm{~cm}$ offset in the steady-state behavior. In contrast, the ABS takes some time to tune its gains itself, but once is achieved, the robot is able to follow the trajectory efficiently despite the weight of the load. In the same way, the NLPD fails to follow the reference. Indeed, this controller has the worst performance compared to all the others methodologies. Again, the introduction of the observer improves the behavior of the NLPD as can be seen in the top of Fig. 7. Concerning the trajectory tracking in yaw, the whole set of controllers show a good performance. The plot of the errors for both tracking tasks is displayed in the middle of Fig. 7, and its numerical representation is shown in Tables 5 and 6. At the bottom of Fig. 7, the evolution of the control inputs is displayed. While the performance of the BS and the ABS controllers are very similar, the ANLPD shows an aggressive behavior compared to the NLPD. Regarding energy consumption, using the BS requires 1.03 time more energy 
than using the ABS for both tracking tests, as illustrated by the ratio below:

$$
I N T_{z}=\frac{I N T_{z}(B S)}{I N T_{z}(A B S)}=1.03 ; I N T_{\psi}=\frac{I N T_{\psi}(B S)}{I N T_{\psi}(A B S)}=1.03
$$

The energy consumption using the ANLPD is 1.12 time the energy consumption using the NLPD for the depth tracking test. For the heading tracking task, the energy consumption employing the ANLPD is 1.09 times the energy consumption using the NLPD. The ratios are computed as:

$$
I N T_{z}=\frac{I N T_{z}(A N L P D)}{I N T_{z}(N L P D)}=1.12 ; I N T_{\psi}=\frac{I N T_{\psi}(A N L P D)}{I N T_{\psi}(N L P D)}=1.09
$$

The plots of the adaption of the observer gains for the BS and NLPD techniques are shown in Figs. 6 and 8 , respectively. The estimation of the disturbance made by the observer using the ABS and ANLPD, respectively, is displayed at the bottom of Fig. 9 .

Remark 9. It is worth to note that in this manuscript, we have considered constant disturbances on the real-time experiments to test the robustness of the proposed controller. However, based on the results of Theorem 1, the algorithm is robust towards bounded time-varying external disturbances theoretically. This scenario will be part of a future research in this topic.

\section{Conclusion}

In this paper, an adaptive disturbance observer based on the extended state observer and high order sliding mode technique has been proposed. The adaptive disturbance observer is introduced into the Backstepping and nonlinear PD controllers to improve the performance of these techniques. The stability analysis for the resulting closed-loop system for trajectory tracking has been addressed. The proposed controller has been implemented for trajectory tracking in depth and yaw motions with the Leonard underwater vehicle and have been compared to the nominal design proposed in our previous works. The real-time experiments results demonstrate the effectiveness, robustness, and improvement of the proposed scheme to uncertainties on the parameters of the system (damping and buoyancy changes) and to external disturbances, as well. In this manuscript, constant disturbances were considered, the robustness of the proposed towards time-varying external disturbances and the improvement of the proposed scheme are part of the future work. 


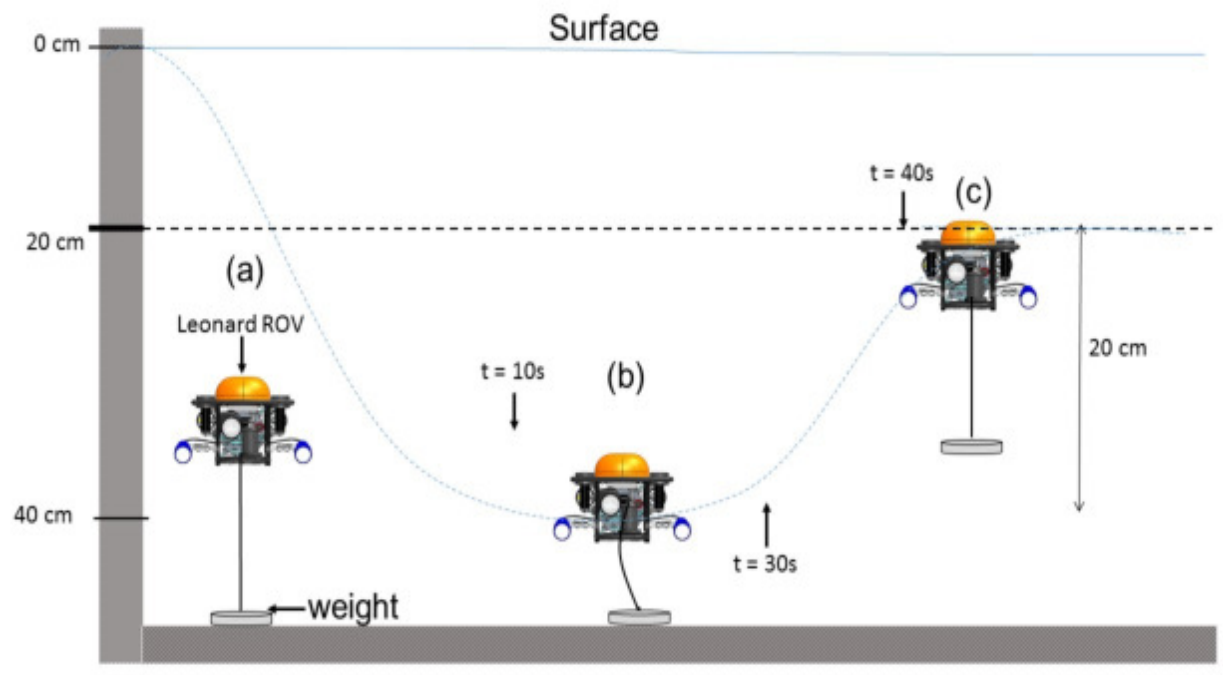

Figure 3: Description of the third scenario. (a) A1 $\mathrm{kg}$ load is attached to the Submarine. When the robot reaches $30 \mathrm{~cm}$, the influence of the weight disappears (b). Finally, the robot comes up again and the influence of the weight acts again on the robot (c).

407

\section{Acknowledgment}

The authors would like to express their gratitude to the anonymous reviewers for the comments to the improvement of the manuscript. The Leonard underwater vehicle has been financed by the European Union (FEDER grant $\mathrm{n}^{\circ}$ 49793) and the Region Occitanie (ARPE Pilot Plus project). The authors thank CONACYT for the scholarship grant (490978). 

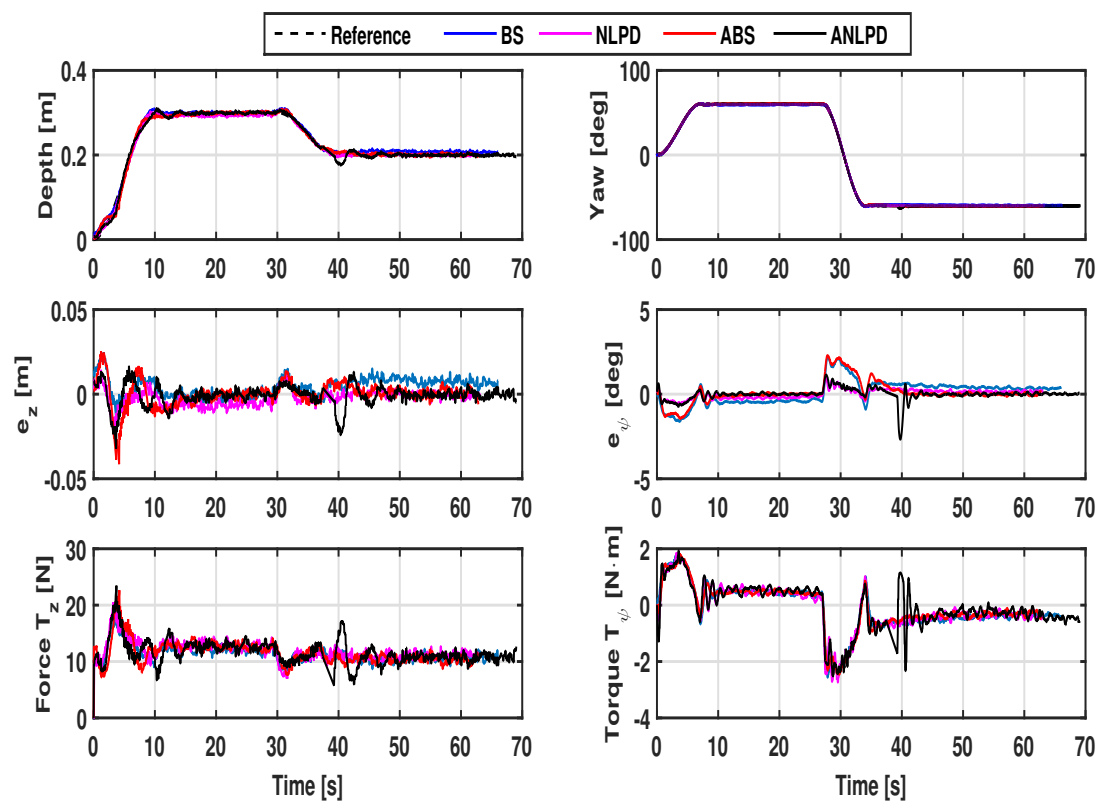

Figure 4: Performance of the NLPD and BS and their adaptive schemes for the depth and yaw tracking trajectory task in the nominal case.
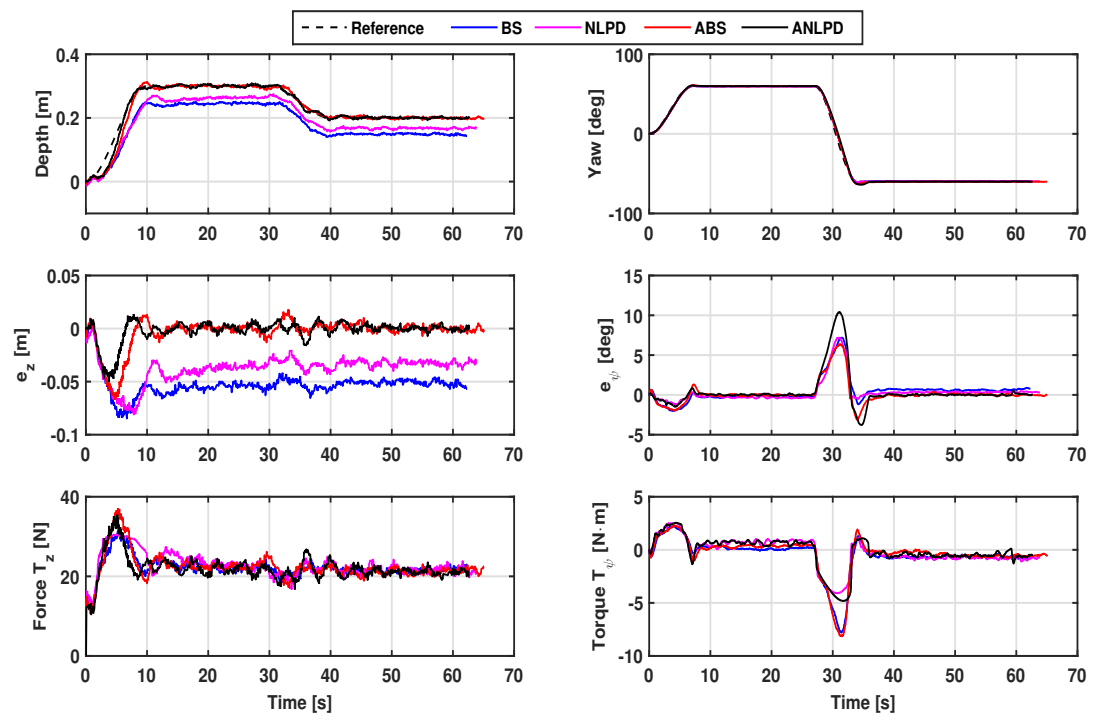

Figure 5: Robustness of the BS and NLPD and their adaptive versions behavior to parametric uncertainties. The floatability of the submarine was increased $+100 \%$, while the damping along z-axis was modify up to $90 \%$ with respect to the nominal case. 

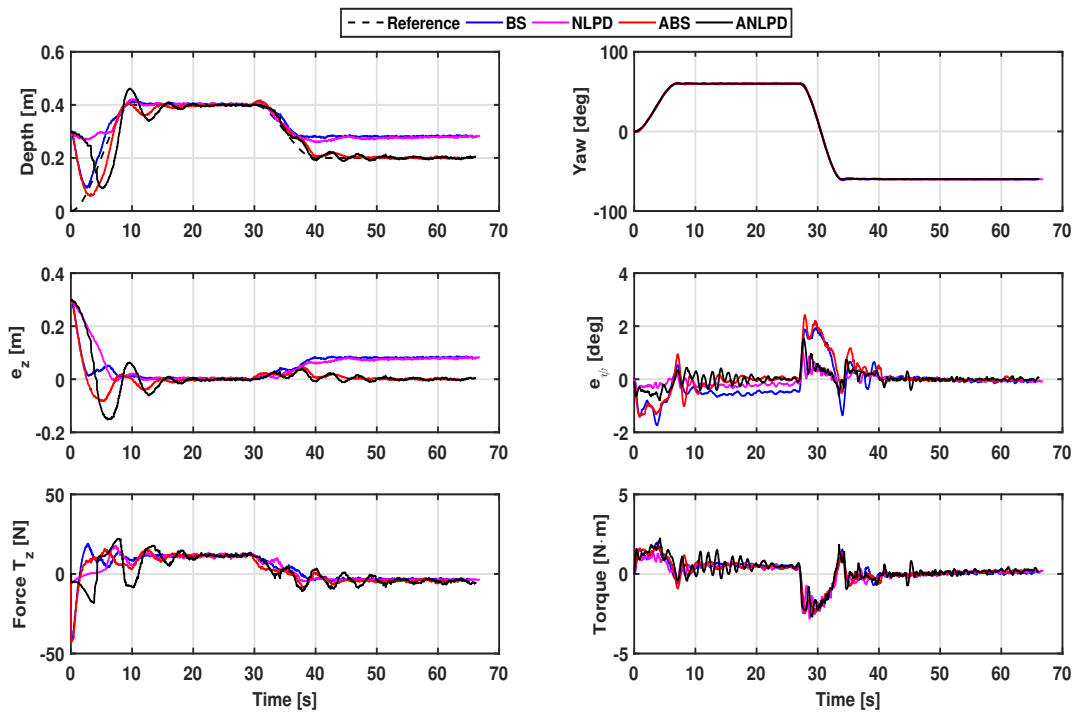

Figure 6: Robustness of the BS, ANLPD and their adaptive versions to external disturbances: Release and recovery of a load.
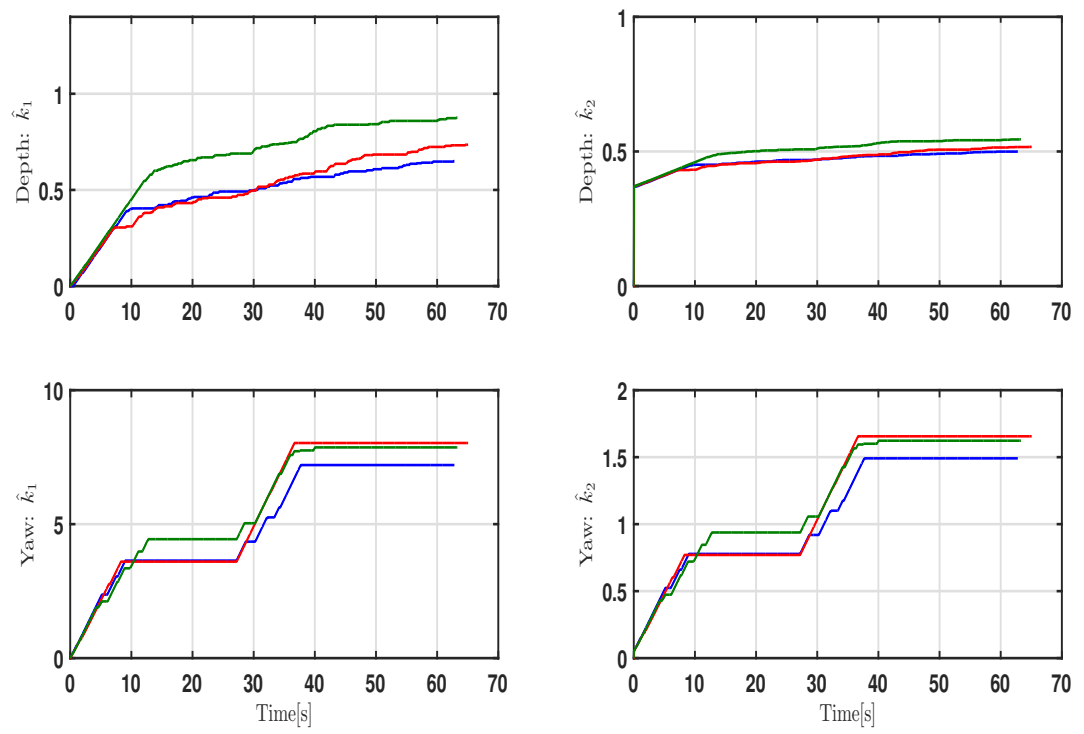

Figure 7: The dynamic gains evolution of the adaptive Backstepping disturbance observer: The nominal case (blue line), scenario 2 (red line) and scenario 3 (green line). 

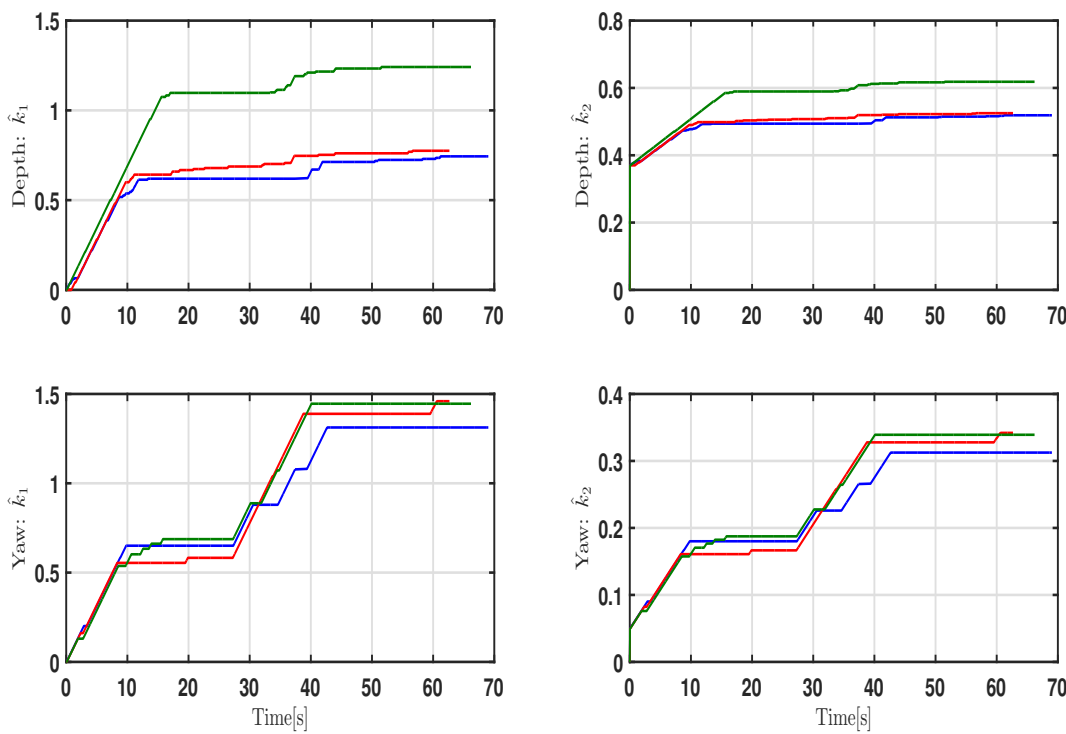

Figure 8: Evolution of the ANLPD disturbance observer gains: Nominal case (blue line), scenario 2 (red line) and scenario 3 (green line).
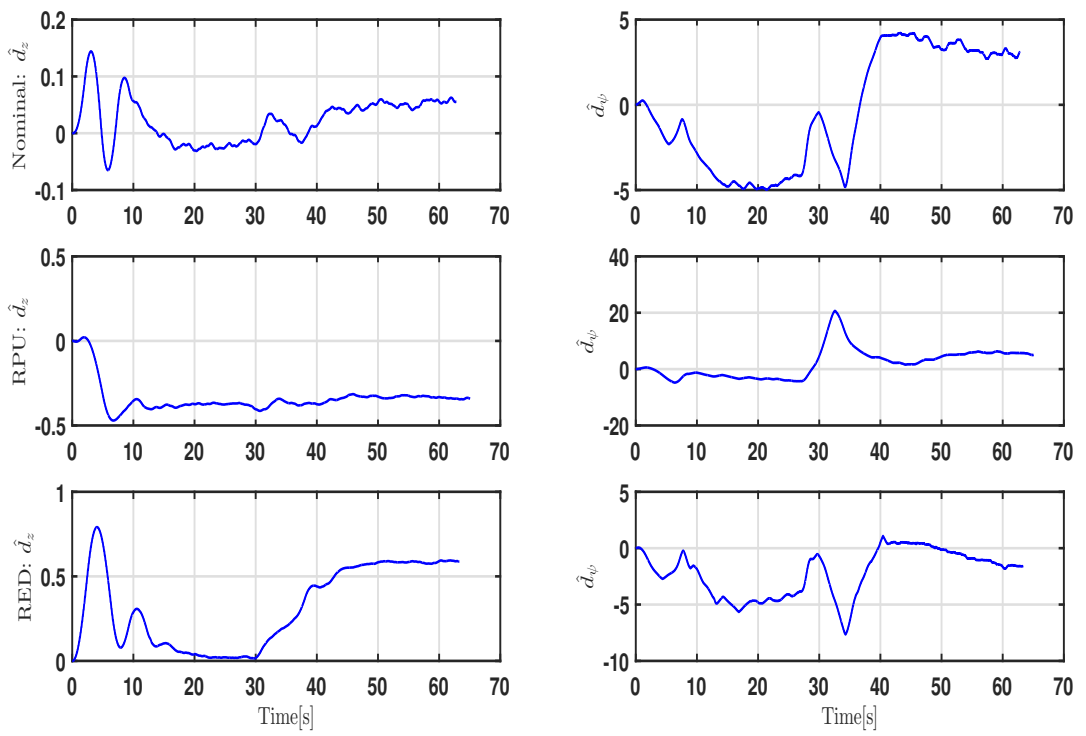

Figure 9: Disturbances estimated by the ABS: Nominal case (upper), robustness towards parametric uncertainties (middle) and robustness towards external disturbances (bottom). 

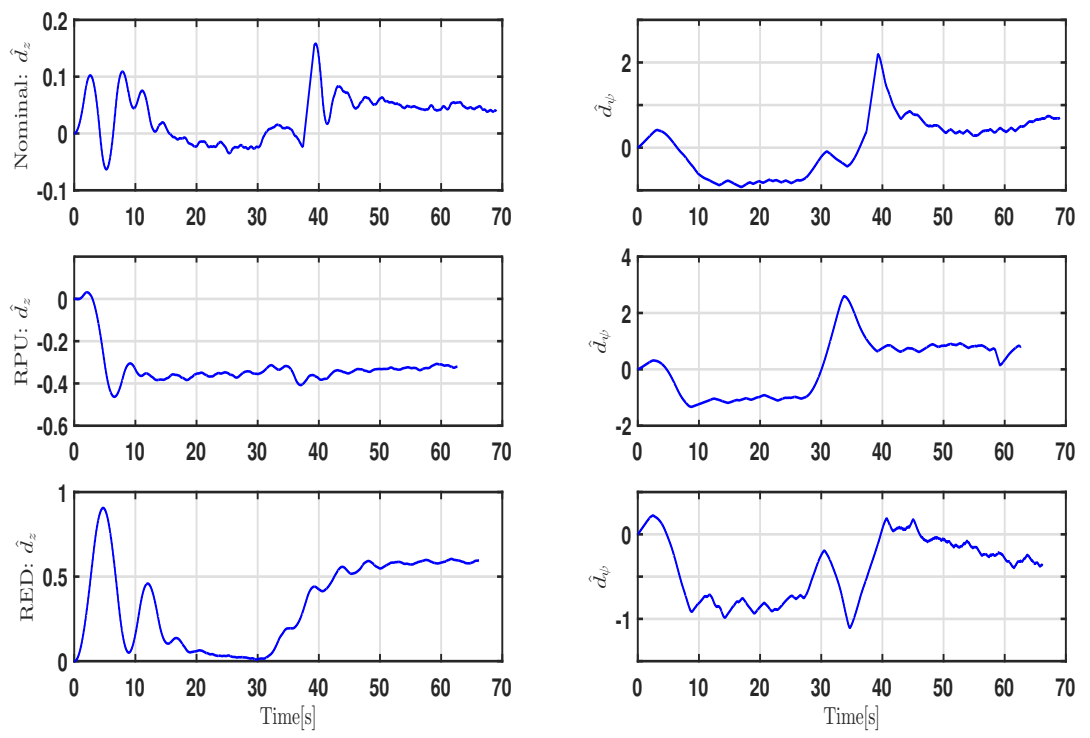

Figure 10: Disturbances estimated by the ANLPD: Nominal case (upper), robustness towards parametric uncertainties (middle) and robustness towards external disturbances (bottom). 


\begin{tabular}{|c|c|c|c|c|}
\hline \multirow{2}{*}{ Case } & \multicolumn{2}{|c|}{ BS } & \multicolumn{2}{|c|}{ ABS } \\
\hline & $R M S E_{z}[m]$ & $R M S E_{\psi}[\operatorname{deg}]$ & $R M S E_{z}[m]$ & $R M S E_{\psi}[\operatorname{deg}]$ \\
\hline Nominal & 0.0043 & 0.1041 & 0.0001 & 0.1071 \\
\hline $\begin{array}{l}\text { Parametric } \\
\text { Uncertainties }\end{array}$ & 0.0530 & 0.4891 & 0.0031 & 0.1487 \\
\hline $\begin{array}{l}\text { External } \\
\text { Disturbances }\end{array}$ & 0.0476 & 0.1210 & 0.0053 & 0.0661 \\
\hline
\end{tabular}

Table 5: Root Mean Square Error for BS and ABS design.

\begin{tabular}{|c|c|c|c|c|}
\hline \multirow{2}{*}{ Case } & \multicolumn{2}{|c|}{ NLPD } & \multicolumn{2}{|c|}{ ANLPD } \\
\hline & $R M S E_{z}[m]$ & $R M S E_{\psi}[\operatorname{deg}]$ & $R M S E_{z}[m]$ & $R M S E_{\psi}[\operatorname{deg}]$ \\
\hline Nominal & 0.0023 & 0.0265 & 0.0007 & 0.0099 \\
\hline $\begin{array}{l}\text { Parametric } \\
\text { Uncertainties }\end{array}$ & 0.0374 & 0.3371 & 0.0018 & 0.4068 \\
\hline $\begin{array}{l}\text { External } \\
\text { Disturbances }\end{array}$ & 0.0522 & 0.0571 & 0.0079 & 0.0170 \\
\hline
\end{tabular}

Table 6: Root Mean Square Error for NLPD and ANLPD designs.

\begin{tabular}{lcccc}
\hline \multirow{2}{*}{ Case } & \multicolumn{2}{c}{ BS } & \multicolumn{2}{c}{ ABS } \\
\cline { 2 - 5 } & $\boldsymbol{I N T}_{\boldsymbol{z}}$ & $\boldsymbol{I N}_{\boldsymbol{\psi}}$ & $\boldsymbol{I N} \boldsymbol{T}_{\boldsymbol{z}}$ & $\boldsymbol{I N \boldsymbol { T } _ { \boldsymbol { \psi } }}$ \\
\hline Nominal & 546 & 33 & 557 & 28 \\
$\begin{array}{l}\text { Parametric } \\
\begin{array}{l}\text { Uncertainties } \\
\text { External } \\
\text { Disturbances }\end{array}\end{array}$ & 1064 & 48 & 1077 & 48 \\
\hline
\end{tabular}

Table 7: Integral control of inputs for BS and ABS designs. 


\begin{tabular}{lcccc}
\hline \multirow{2}{*}{ Case } & \multicolumn{2}{c}{ NLPD } & \multicolumn{2}{c}{ ANLPD $^{\prime}$} \\
\cline { 2 - 5 } & $\boldsymbol{I N T}_{\boldsymbol{z}}$ & $\boldsymbol{I N T}_{\boldsymbol{\psi}}$ & $\boldsymbol{I N \boldsymbol { T } _ { \boldsymbol { z } }}$ & $\boldsymbol{I N \boldsymbol { N } _ { \boldsymbol { \psi } }}$ \\
\hline Nominal & 567 & 25 & 557 & 29 \\
$\begin{array}{l}\text { Parametric } \\
\text { Uncertainties }\end{array}$ & 1099 & 51 & 1054 & 52 \\
$\begin{array}{l}\text { External } \\
\text { Disturbances }\end{array}$ & 361 & 32 & 405 & 35 \\
\hline
\end{tabular}

Table 8: Integral control of inputs for NLPD and ANLPD designs. 


\section{References}

[1] S. Zhao, J. Yuh, Experimental study on advanced underwater robot control, IEEE transactions on robotics 21 (2005) 695-703.

[2] B. Jalving, The ndre-auv flight control system, IEEE Journal of Oceanic Engineering 19 (1994) 497-501.

[3] P. Herman, Decoupled pd set-point controller for underwater vehicles, Ocean Engineering 36 (2009) 529-534.

[4] T. I. Fossen, Guidance and control of ocean vehicles, John Wiley \& Sons Inc, 1994.

[5] T. T. J. Prestero, Verification of a six-degree of freedom simulation model for the REMUS autonomous underwater vehicle, Ph.D. thesis, Massachusetts institute of technology, 2001.

[6] L. Moreira, C. G. Soares, $\mathrm{H}_{2}$ and $\mathrm{H}_{\infty}$ designs for diving and course control of an autonomous underwater vehicle in presence of waves, IEEE Journal of Oceanic engineering 33 (2008) 69-88.

[7] R. da Silva Tchilian, E. Rafikova, S. A. Gafurov, M. Rafikov, Optimal control of an underwater glider vehicle, Procedia Engineering 176 (2017) 732-740.

[8] L. G. García-Valdovinos, T. Salgado-Jiménez, M. Bandala-Sánchez, L. Nava-Balanzar, R. Hernández-Alvarado, J. A. Cruz-Ledesma, Modelling, design and robust control of a remotely operated underwater vehicle, International Journal of Advanced Robotic Systems 11 (2014) 1.

[9] Z. H. Ismail, V. W. Putranti, Second order sliding mode control scheme for an autonomous underwater vehicle with dynamic region concept, Mathematical Problems in Engineering 2015 (2015).

[10] J. Kim, H. Joe, S.-c. Yu, J. S. Lee, M. Kim, Time-delay controller design for position control of autonomous underwater vehicle under disturbances, IEEE Transactions on Industrial Electronics 63 (2016) 10521061. 
[11] Y. Shtessel, M. Taleb, F. Plestan, A novel adaptive-gain supertwisting sliding mode controller: Methodology and application, Automatica 48 (2012) 759-769.

[12] M. Krstic, I. Kanellakopoulos, P. V. Kokotovic, et al., Nonlinear and adaptive control design, volume 222, Wiley New York, 1995.

[13] B. Sun, D. Zhu, S. X. Yang, A bioinspired filtered backstepping tracking control of 7000-m manned submarine vehicle., IEEE Trans. Industrial Electronics 61 (2014) 3682-3693.

[14] J. Guerrero, J. Torres, E. Antonio, E. Campos, Autonomous underwater vehicle robust path tracking: Generalized super-twisting algorithm and block backstepping controllers, Journal of Control Engineering and Applied Informatics 20 (2018) 51-63.

[15] L. Zhang, L. Zhang, S. Liu, J. Zhou, C. Papavassiliou, Low-level control technology of micro autonomous underwater vehicle based on intelligent computing, Cluster Computing (2018) 1-12.

[16] P. Sarhadi, A. R. Noei, A. Khosravi, Model reference adaptive pid control with anti-windup compensator for an autonomous underwater vehicle, Robotics and Autonomous Systems 83 (2016) 87-93.

[17] M. H. Khodayari, S. Balochian, Modeling and control of autonomous underwater vehicle (auv) in heading and depth attitude via self-adaptive fuzzy pid controller, Journal of Marine Science and Technology 20 (2015) $559-578$.

[18] E. Campos, A. Chemori, V. Creuze, J. Torres, R. Lozano, Saturation based nonlinear depth and yaw control of underwater vehicles with stability analysis and real-time experiments, Mechatronics 45 (2017) 49-59.

[19] J. Guerrero, J. Torres, V. Creuze, A. Chemori, E. Campos, Saturation based nonlinear pid control for underwater vehicles: Design, stability analysis and experiments, Mechatronics 61 (2019) 96-105.

[20] R. Cui, L. Chen, C. Yang, M. Chen, Extended state observer-based integral sliding mode control for an underwater robot with unknown disturbances and uncertain nonlinearities, IEEE Transactions on Industrial Electronics 64 (2017) 6785-6795. 
[21] J. A. Moreno, A linear framework for the robust stability analysis of a generalized super-twisting algorithm, in: Electrical Engineering, Computing Science and Automatic Control, CCE, 2009 6th International Conference on, IEEE, pp. 1-6.

[22] J. Guerrero, J. Torres, V. Creuze, A. Chemori, Trajectory tracking for autonomous underwater vehicle: An adaptive approach, Ocean Engineering 172 (2019) 511-522.

[23] S. of Naval Architects, M. E. U. Technical, R. C. H. Subcommittee, Nomenclature for Treating the Motion of a Submerged Body Through a Fluid: Report of the American Towing Tank Conference, Technical and research bulletin, Society of Naval Architects and Marine Engineers, 1950 .

[24] T. I. Fossen, Marine control systems: guidance, navigation and control of ships, rigs and underwater vehicles, 2002.

[25] J. C. Kinsey, R. M. Eustice, L. L. Whitcomb, A survey of underwater vehicle navigation: Recent advances and new challenges, in: IFAC Conference of Manoeuvering and Control of Marine Craft, volume 88, pp. $1-12$.

[26] S. Soylu, B. J. Buckham, R. P. Podhorodeski, A chattering-free slidingmode controller for underwater vehicles with fault-tolerant infinity-norm thrust allocation, Ocean Engineering 35 (2008) 1647-1659.

[27] J. Han, A class of extended state observers for uncertain systems, Control and decision 10 (1995) 85-88.

[28] J. Han, Auto-disturbance rejection control and its applications, Control and decision 13 (1998) 19-23.

[29] J. Guerrero, J. Torres, V. Creuze, A. Chemori, Observation-based nonlinear proportional-derivative control for robust trajectory tracking for autonomous underwater vehicles, IEEE Journal of Oceanic Engineering (2019).

[30] F. Plestan, Y. Shtessel, V. Bregeault, A. Poznyak, New methodologies for adaptive sliding mode control, International journal of control 83 (2010) 1907-1919. 
[31] A. Levant, Sliding order and sliding accuracy in sliding mode control, International journal of control 58 (1993) 1247-1263.

[32] Y. Zhang, P. Yan, Sliding mode disturbance observer-based adaptive integral backstepping control of a piezoelectric nano-manipulator, Smart Materials and Structures 25 (2016) 125011. 